\title{
Fixed point theorems for sum operator with parameter
}

\author{
Nan Zhang ${ }^{1}$, Lingling Zhang ${ }^{1,2^{*}}$, Bibo Zhou ${ }^{1}$ and Huimin Tian ${ }^{1}$
}

\author{
Correspondence: tyutzll@126.com \\ 1 Department of Mathematics, \\ Taiyuan University of Technology, \\ Taiyuan, China \\ ${ }^{2}$ State Key Laboratory of Explosion \\ Science and Technology, Beijing \\ Institute of Technology, Beijing, \\ China
}

\begin{abstract}
This paper develops some new existence and uniqueness theorems of a fixed point for a class of sum operator equations with parameter

$\lambda_{1} A(x, x)+\lambda_{2} B(x, x)+\lambda_{3} C x+\lambda_{4} D x=x$, where $A, B$ are two mixed monotone operators,

$C$ is an increasing operator, $D$ is a decreasing operator. In the case of positive parameters, the results obtained in this paper extend many existing conclusions in the field of study. Furthermore, by using the properties of Green's function and the above fixed point theorems of sum operator, the unique positive solution a class of fractional differential equations with integro-differential boundary value conditions is given. Application of the results to the study of fractional differential equations is also given in the article.
\end{abstract}

Keywords: Mixed monotone operators; Concave-convex operator; Operator equation; Positive solution; Fixed point theorem; Fractional differential equation

\section{Introduction}

This paper discusses the existence and uniqueness of solution for a class of operator equation

$$
\lambda_{1} A(x, x)+\lambda_{2} B(x, x)+\lambda_{3} C x+\lambda_{4} D x=x,
$$

where $A, B$ are two mixed monotone operators, $C$ is an increasing operator, $D$ is a decreasing operator, $\lambda_{i}>0(i=1,2,3,4)$ and satisfies the following conditions:

Situation 1:

1. $A\left(\lambda x, \lambda^{-1} y\right) \geq \varphi(\lambda) A(x, y), \varphi(\lambda) \in(\lambda, 1], \forall \lambda \in(0,1), x, y \in P$;

2. for any fixed $y \in P, B(\cdot, y): P \rightarrow P$ is concave; for any $x \in P, B(x, \cdot): P \rightarrow P$ is convex;

3. $C: P \rightarrow P$ is increasing sub-homogeneous.

4. $D\left(\lambda^{-1} y\right) \geq \lambda D y, \forall \lambda \in(0,1), y \in P$.

Situation 2:

1. $A\left(\lambda x, \lambda^{-1} y\right) \geq \lambda A(x, y), \forall \lambda \in(0,1), x, y \in P$;

2. for any fixed $y \in P, B(\cdot, y): P \rightarrow P$ is concave; for any $x \in P, B(x, \cdot): P \rightarrow P$ is convex;

3. $C(\lambda x) \geq \varphi(\lambda) C x, \varphi(\lambda) \in(\lambda, 1], \forall \lambda \in(0,1), x, y \in P$;

4. $D\left(\lambda^{-1} y\right) \geq \lambda D y, \forall \lambda \in(0,1), y \in P$.

(c) The Author(s) 2020. This article is licensed under a Creative Commons Attribution 4.0 International License, which permits use, sharing, adaptation, distribution and reproduction in any medium or format, as long as you give appropriate credit to the original author(s) and the source, provide a link to the Creative Commons licence, and indicate if changes were made. The images or other third party material in this article are included in the article's Creative Commons licence, unless indicated otherwise in a credit line to the material. If material is not included in the article's Creative Commons licence and your intended use is not permitted by statutory regulation or exceeds the permitted use, you will need to obtain permission directly from the copyright holder. To view a copy of this licence, visit http://creativecommons.org/licenses/by/4.0/. 
In fact, the operator equation $\lambda_{1} A(x, x)+\lambda_{2} B(x, x)+\lambda_{3} C x+\lambda_{4} D x=x$ generalizes and improves many articles.

When the parameter $\lambda_{3}=1, \lambda_{i}=0(i=1,2,4)$, situation 2 can be reduced to the case that an increasing operator $C$ meets $C(t x) \geq t^{\alpha(t)} C x, \alpha(t) \in(0,1), \forall t \in(0,1), x \in P_{h}$. Obviously, it is the result of the paper [1].

When the parameter $\lambda_{1}=1, \lambda_{i}=0(i=2,3,4)$, there is $A\left(\lambda x, \lambda^{-1} y\right) \geq \varphi(\lambda) A(x, y), \varphi(\lambda) \in$ $(\lambda, 1], \forall \lambda \in(0,1), x, y \in P$ in situation 1 . In [2], we can see that the mixed monotone operator $A$ meets the same properties.

When the parameter $\lambda_{1}=1, \lambda_{2}=0, \lambda_{3}=1, \lambda_{4}=0$, we deduce from situation 1 that Theorem 2.1 of [3] is established and from situation 2 that Theorem 2.4 of [3] holds.

When the parameter $\lambda_{1}=1, \lambda_{2}=0, \lambda_{3}=1, \lambda_{4}=1$, we derive Theorem 3.1 of [4] from situation 1 and Theorem 3.8 of [4] from situation 1 .

When the parameter $\lambda_{1}=1, \lambda_{2}=1, \lambda_{3}=1, \lambda_{4}=0$, the conditions of situation 1 are the same as those of Theorem 3.1 in [5], thus they can get same conclusions.

The theory of nonlinear operators plays a significant role in modern mathematics and there are many excellent results (see [1-7]). Inspired by the these paper, we want to make some contributions to this field. As one of the applications of operator theory, fractional differential equations have attracted much attention by many researchers as a result of a myriad of their applications in many engineering and scientific disciplines, such as mechanics, biomedicine, physics, and so on, see [8-15] and the references therein. Besides, there are some methods such as comparison theorem, the monotone iterative technique, the method of lower and upper solutions, Leray-Schauder theory, Krasnoselskii's fixed point theorems, and some other fixed point theorems in cones. They play an irreplaceable role in the existence, uniqueness, and multiplicity of positive solutions for fractional differential equations [16-22]. In recent decades, more and more fractional differential equations are solved based on the nonlinear operators theory [23-25]. In this paper, we make use of the new operator equation theory to investigate a class of new fractional differential equations.

The characteristic features of this paper are displayed as follows. Firstly, comparatively speaking, we generalize the results of the above article. Secondly, there are seldom investigated operator equations with parameters. A class of new operator equations with four operators $\lambda_{1} A(x, x)+\lambda_{2} B(x, x)+\lambda_{3} C x+\lambda_{4} D x=x$ is studied, and the fixed point theorem of this sum-type operator is obtained. We gain the existence and uniqueness solution of the operator equation, and construct two iterative sequences to uniformly approximate this solution in the fixed point theorem. Thirdly, by using the new results, we study a class of new fractional differential equations and get some great conclusions. Fourthly, some concrete examples are given to illustrate the main ideas.

The main body of this paper is organized as follows. In Sect. 2, we review the theory and results of fractional calculus and some definitions, notations in Banach space. In Sect. 3, a class of fixed point theorem is presented. In Sect. 4, by the theorem of Sect. 2, a kind of fractional differential equation is studied and some examples to illustrative our work are presented.

\section{Preliminaries}

In this section, since all the work is in the Banach space, a brief review about the Banach space and relevant contents is given for the reader's convenience; it includes some defini- 
tions, lemmas, and basic results. These will be used in the following proofs of our theorem. For more details, we refer the reader to [8-12].

Let $(E,\|\cdot\|)$ be a real Banach space. A nonempty closed convex set $P \subset E$ is called a cone if the following hold:

(i) If $x \in P, \lambda \geq 0$, then $\lambda x \in P$;

(ii) If $x \in P$ and $-x \in P$, then $x=\theta$, where $\theta$ is the zero element of $E$.

There is partially ordered by a cone $P \subset E, x \leq y, x, y \in E \Leftrightarrow y-x \in P$, in which, if $x \leq y$ and $x \neq y$, we denote $x<y$ or $y>x$.

If $\dot{P}=\{x \in P \mid x$ is an interior point of $P\}$ is nonempty, cone $P$ is said to be solid. Then, we call $P$ normal if there exists a constant $N>0$ such that, for $\forall x, y \in E, \theta \leq x \leq y$, there is $\|x\| \leq N\|y\|$, where $N$ is called the normality constant of $P$. For $\forall x, y \in E$, the denotation $x \sim y$ means that there exist $\lambda, \mu>0$ such that $\lambda x \leq y \leq \mu x$. Clearly, $\sim$ is an equivalence relation. Given $h>\theta$, and denoting the set $P_{h}=\{x \in E \mid x \sim h\}$, there is $P_{h} \subset P$.

Definition 2.1 ([8]) $A: P \times P \rightarrow P$ is called a mixed monotone operator if $A(x, y)$ is increasing in $x$ and decreasing in $y$, i.e., $u_{i}, v_{i}(i=1,2) \in P, u_{1} \leq u_{2}, v_{1} \geq v_{2}$ implies $A\left(u_{1}, v_{1}\right) \leq A\left(u_{2}, v_{2}\right)$. Element $x \in P$ is called a fixed point of $A$ if $A(x, x)=x$.

Definition 2.2 $([9,10])$ An operator $A: E \rightarrow E$ is said to be homogeneous if it satisfies

$$
A(t x)=t A x, \quad \forall t>0, x \in E .
$$

An operator $A: P \rightarrow P$ is said to be sub-homogeneous if it satisfies

$$
A(t x) \geq t A x, \quad \forall t \in(0,1), x \in P .
$$

Definition 2.3 $([9,10])$ Let $D=P$ or $D=\dot{P}$ and a real number $\alpha \in[0,1)$. An operator $A: D \rightarrow D$ is said to be $\alpha$-concave if it satisfies

$$
A(t x) \geq t^{\alpha} A x, \quad \forall t \in(0,1), x \in D .
$$

Definition 2.4 ([11]) Let $D$ be a convex subset in $E$. An operator $A: D \rightarrow E$ is called a convex operator if, for $\forall x, y \in D$ with $y \leq x$ and every $t \in[0,1]$,

$$
A(t x+(1-t) y) \leq t A x+(1-t) A y
$$

$A: D \rightarrow E$ is called a concave operator if

$$
A(t x+(1-t) y) \geq t A x+(1-t) A y .
$$

\section{Main results}

In this section, we use the definitions, notations of section Preliminaries to investigate some new fixed point theorem. Furthermore, we can obtain the following sufficient conditions of existence and uniqueness of positive solutions for the operator equation $\lambda_{1} A(x, x)+\lambda_{2} B(x, x)+\lambda_{3} C x+\lambda_{4} D x=x$. 
Theorem 3.1 There are four operators $A, B, C, D$, where $A, B: P \times P \rightarrow P$ are two mixed monotone operators, $C: P \rightarrow P$ is an increasing sub-homogeneous operator, $D: P \rightarrow P$ is a decreasing operator, and if the following conditions are satisfied:

$\left(A_{1}\right)$ There exists $\varphi(\lambda) \in(\lambda, 1]$ such that

$$
A\left(\lambda x, \lambda^{-1} y\right) \geq \varphi(\lambda) A(x, y), \quad D\left(\lambda^{-1} y\right) \geq \lambda D y, \quad \forall \lambda \in(0,1), x, y \in P
$$

$\left(A_{2}\right)$ For any fixed $y \in P, B(\cdot, y): P \rightarrow P$ is concave; for any $x \in P, B(x, \cdot): P \rightarrow P$ is convex;

$\left(A_{3}\right)$ There exists $\frac{1}{2} \leq \widetilde{c} \leq 1$ such that $B(\theta, l h) \geq \widetilde{c} B(l h, \theta), l \geq 1$;

$\left(A_{4}\right)$ There exists $h \in P$ with $h \neq \theta$ such that $A(h, h), B(h, h), C h, D h \in P_{h}$;

$\left(A_{5}\right)$ There exists $\delta>0$ such that $\left[\lambda_{2} B(x, y)+\lambda_{3} C x+\lambda_{4} D y\right] \leq \delta \lambda_{1} A(x, y), \forall x, y \in P_{h}$.

Then the following conclusions hold:

(C1) $A, B: P_{h} \times P_{h} \rightarrow P_{h}, C, D: P_{h} \rightarrow P_{h}$;

(C2) There exist $u_{0}, v_{0} \in P_{h}, r \in(0,1)$ such that $r v_{0} \leq u_{0}<v_{0}$,

$$
\begin{aligned}
u_{0} & \leq \lambda_{1} A\left(u_{0}, v_{0}\right)+\lambda_{2} B\left(u_{0}, v_{0}\right)+\lambda_{3} C u_{0}+\lambda_{4} D v_{0} \\
& \leq \lambda_{1} A\left(v_{0}, u_{0}\right)+\lambda_{2} B\left(v_{0}, u_{0}\right)+\lambda_{3} C v_{0}+\lambda_{4} D u_{0} \leq v_{0} ;
\end{aligned}
$$

(C3) The operator equation $\lambda_{1} A(x, x)+\lambda_{2} B(x, x)+\lambda_{3} C x+\lambda_{4} D x=x$ has a unique solution $x^{*} \in P_{h}$;

(C4) Constructing the iterative sequences as follows:

$$
\begin{aligned}
& x_{n}=\lambda_{1} A\left(x_{n-1}, y_{n-1}\right)+\lambda_{2} B\left(x_{n-1}, y_{n-1}\right)+\lambda_{3} C x_{n-1}+\lambda_{4} D y_{n-1}, \\
& y_{n}=\lambda_{1} A\left(y_{n-1}, x_{n-1}\right)+\lambda_{2} B\left(y_{n-1}, x_{n-1}\right)+\lambda_{3} C y_{n-1}+\lambda_{4} D x_{n-1}, \quad n=1,2, \ldots,
\end{aligned}
$$

for any initial values $x_{0}, y_{0} \in P_{h}$, we have

$$
x_{n} \rightarrow x^{*}, \quad y_{n} \rightarrow x^{*}, \quad \text { as } n \rightarrow \infty .
$$

Proof Define the operator $T=\lambda_{1} A+\lambda_{2} B+\lambda_{3} C+\lambda_{4} D$ by

$$
T(x, y)=\lambda_{1} A(x, y)+\lambda_{2} B(x, y)+\lambda_{3} C x+\lambda_{4} D y, \quad \forall x, y \in P .
$$

Firstly, we show that $T: P_{h} \times P_{h} \rightarrow P_{h}$. The proof of $A, B: P_{h} \times P_{h} \rightarrow P_{h}, C, D: P_{h} \rightarrow P_{h}$ is expanded. By $A\left(\lambda x, \lambda^{-1} y\right) \geq \varphi(\lambda) A(x, y)$ of $\left(A_{1}\right)$, we deduce

$$
A\left(\lambda^{-1} x, \lambda y\right) \leq \varphi(\lambda)^{-1} A(x, y) .
$$

Furthermore, the condition $A(h, h) \in P_{h}$ of $\left(A_{4}\right)$ shows that there exist positive constants $\overline{u_{1}}, \overline{v_{1}}$ such that

$$
\overline{u_{1}} h \leq A(h, h) \leq \overline{v_{1}} h .
$$

For $\forall x, y \in P_{h}$, we can find two sufficiently small numbers $c_{1}, c_{2} \in(0,1)$ such that

$$
c_{1} h \leq x \leq c_{1}^{-1} h, \quad c_{2} h \leq y \leq c_{2}^{-1} h .
$$


Let $c=\min \left\{c_{1}, c_{2}\right\}$, then by $(1)-(4)$, we get

$$
\begin{aligned}
& A(x, y) \leq A\left(c_{1}^{-1} h, c_{2} h\right) \leq A\left(c^{-1} h, c h\right) \leq \varphi(c)^{-1} A(h, h) \leq \varphi(c)^{-1} \overline{v_{1}} h, \\
& A(x, y) \geq A\left(c_{1} h, c_{2}^{-1} h\right) \geq A\left(c h, c^{-1} h\right) \geq \varphi(c) A(h, h) \geq \varphi(c) \overline{u_{1}} h .
\end{aligned}
$$

Thus, $A: P_{h} \times P_{h} \rightarrow P_{h}$ is proved. Then, due to $\left(A_{2}\right)$, for any $\lambda \in(0,1)$, there is

$$
B(x, y)=B\left(x, \lambda \lambda^{-1} y+(1-\lambda) \theta\right) \leq \lambda B\left(x, \lambda^{-1} y\right)+(1-\lambda) B(x, \theta),
$$

thus, $\lambda B\left(x, \lambda^{-1} y\right) \geq B(x, y)-(1-\lambda) B(x, \theta)$. Subsequently, we can find a sufficiently large $l$ such that $x, y, \lambda^{-1} y \leq l h$. Combining with Definition 2.4 and from the condition of $\left(A_{2}\right)-$ $\left(A_{3}\right)$, we know that

$$
\begin{aligned}
B\left(\lambda x, \lambda^{-1} y\right) & =B\left(\lambda x+(1-\lambda) \theta, \lambda^{-1} y\right) \\
& \geq \lambda B\left(x, \lambda^{-1} y\right)+(1-\lambda) B\left(\theta, \lambda^{-1} y\right) \\
& \geq B(x, y)-(1-\lambda) B(x, \theta)+(1-\lambda) B\left(\theta, \lambda^{-1} y\right) \\
& \geq B(x, y)+(1-\lambda)(B(\theta, l h)-B(l h, \theta)) \\
& \geq B(x, y)+(1-\lambda)\left[B(\theta, l h)-\frac{1}{\widetilde{c}} B(\theta, l h)\right] \\
& \geq\left[1+(1-\lambda)\left(1-\frac{1}{\widetilde{c}}\right)\right] B(x, y) \\
& =\left[\left(2-\frac{1}{\widetilde{c}}\right)+\left(\frac{1}{\widetilde{c}}-1\right) \lambda\right] B(x, y) \\
& \geq \lambda B(x, y),
\end{aligned}
$$

that is,

$$
B\left(\lambda x, \lambda^{-1} y\right) \geq \lambda B(x, y)
$$

Then we gain

$$
B\left(\lambda^{-1} x, \lambda y\right) \leq \lambda^{-1} B(x, y), \quad \lambda \in(0,1) .
$$

From the condition $B(h, h) \in P_{h}$, there exist two positive constants $\overline{u_{2}}, \overline{v_{2}}$ such that $\overline{u_{2}} h \leq$ $B(h, h) \leq \overline{v_{2}} h$. For any $x, y \in P_{h}$,

$$
\begin{aligned}
& B(x, y) \leq B\left(c_{1}^{-1} h, c_{2} h\right) \leq B\left(c^{-1} h, c h\right) \leq c^{-1} B(h, h) \leq c^{-1} \overline{v_{2}} h, \\
& B(x, y) \geq B\left(c_{1} h, c_{2}^{-1} h\right) \geq B\left(c h, c^{-1} h\right) \geq c B(h, h) \geq c \overline{u_{2}} h .
\end{aligned}
$$

Thus, $B: P_{h} \times P_{h} \rightarrow P_{h}$ holds. Since $C h, D h \in P_{h}$,

$$
\overline{u_{3}} h \leq C h \leq \overline{v_{3}} h, \quad \overline{u_{4}} h \leq D h \leq \overline{v_{4}} h,
$$


where $\overline{u_{i}}, \overline{v_{i}}(i=3,4)$ are positive constants. By the properties of operators $C, D$, for any $\lambda \in(0,1), x, y \in P$, there is

$$
C(t x) \geq t C x, \quad C\left(t^{-1} x\right) \leq t^{-1} C x, \quad D\left(t^{-1} x\right) \geq t D x, \quad D(t x) \leq t^{-1} D x .
$$

Using similar processes, we have

$$
\begin{aligned}
& C x \leq C\left(c_{1}^{-1} h\right) \leq C\left(c^{-1} h\right) \leq c^{-1} C h \leq c^{-1} \overline{v_{3}} h, \\
& C x \geq C\left(c_{1} h\right) \geq C(c h) \geq c C h \geq c \overline{u_{3}} h, \\
& D y \leq D\left(c_{2} h\right) \leq D(c h) \leq c^{-1} D h \leq c^{-1} \overline{v_{4}} h, \\
& D y \geq D\left(c_{2}^{-1} h\right) \geq D\left(c^{-1} h\right) \geq c D h \geq c \overline{u_{4}} h,
\end{aligned}
$$

which shows that $C, D: P_{h} \rightarrow P_{h}$. From the above deduction, we have

$$
\begin{aligned}
& \left(\lambda_{1} \varphi(c) \overline{u_{1}}+\lambda_{2} c \overline{u_{2}}+\lambda_{3} c \overline{u_{3}}+\lambda_{4} c \overline{u_{4}}\right) h \\
& \quad \leq T(x, y)=\lambda_{1} A(x, y)+\lambda_{2} B(x, y)+\lambda_{3} C x+\lambda_{4} D y \\
& \quad \leq\left(\lambda_{1} \varphi(c)^{-1} \overline{v_{1}}+\lambda_{2} c^{-1} \overline{v_{2}}+\lambda_{3} c^{-1} \overline{v_{3}}+\lambda_{4} c^{-1} \overline{v_{4}}\right) h
\end{aligned}
$$

i.e., $T: P_{h} \times P_{h} \rightarrow P_{h}$, and then we can get $T(h, h) \in P_{h}$.

Secondly, we demonstrate that there exists $\eta(t, x, y) \in(t, 1]$ such that

$$
T\left(t x, t^{-1} y\right) \geq \eta(t, x, y) T(x, y), \quad \forall t \in(0,1), x, y \in P_{h} .
$$

Combining with (1), (5) and the properties of operator $C, D$, we have

$$
\begin{aligned}
T\left(t x, t^{-1} y\right) & =\lambda_{1} A\left(t x, t^{-1} y\right)+\lambda_{2} B\left(t x, t^{-1} y\right)+\lambda_{3} C(t x)+\lambda_{4} D\left(t^{-1} y\right) \\
& \geq \lambda_{1} \varphi(t) A(x, y)+\lambda_{2} t B(x, y)+\lambda_{3} t C x+\lambda_{4} t D y \\
& =\varphi(t) \lambda_{1} A(x, y)+t\left[\lambda_{2} B(x, y)+\lambda_{3} C x+\lambda_{4} D y\right] .
\end{aligned}
$$

Owing to $A, B: P_{h} \times P_{h} \rightarrow P_{h}, C, D: P_{h} \rightarrow P_{h}$, there is $\lambda_{1} A(x, y) \sim \lambda_{2} B(x, y)+\lambda_{3} C x+\lambda_{4} D y$. Define $K\left\{\frac{x}{y}\right\}=\inf \{k \in R \mid x \leq k y\}$, and let

$$
J(x, y)=K\left(\frac{\lambda_{2} B(x, y)+\lambda_{3} C x+\lambda_{4} D y}{\lambda_{1} A(x, y)}\right),
$$

we can easily get $J(x, y) \leq \delta$ by $\left(A_{5}\right)$. Next, considering a function $\bar{h}(s)=\frac{\varphi(t)+J(x, y) t}{U(x, y)+1) s}$, it shows that $h$ is continuous and strictly decreasing about $s$. Due to $\varphi(t)>t$, there is

$$
\bar{h}\left(\frac{\delta t+\varphi(t)}{\delta+1}\right)=\frac{\varphi(t)+J(x, y) t}{(J(x, y)+1) \frac{\delta t+\varphi(t)}{\delta+1}}>1, \quad \bar{h}(\varphi(t))=\frac{\varphi(t)+J(x, y) t}{(J(x, y)+1) \varphi(t)}<1 .
$$

Because of the monotone decreasing of $\bar{h}$, we can get that there exist $\eta(t, x, y) \in\left(\frac{\delta t+\varphi(t)}{\delta+1}\right.$, $\varphi(t)) \subset(t, 1]$,

$$
\bar{h}(\eta(t, x, y))=\frac{\varphi(t)+J(x, y) t}{(J(x, y)+1) \eta(t, x, y)}=1,
$$


thus, $J(x, y)=\frac{\varphi(t)-\eta(t, x, y)}{\eta(t, x, y)-t}$. By the expression of $K, J$, there is

$$
\lambda_{2} B(x, y)+\lambda_{3} C x+\lambda_{4} D y \leq \frac{\varphi(t)-\eta(t, x, y)}{\eta(t, x, y)-t} \lambda_{1} A(x, y), \quad \forall t \in(0,1), x, y \in P_{h} .
$$

Thus, we obtain

$$
\begin{aligned}
T\left(t x, t^{-1} y\right) \geq & \varphi(t) \lambda_{1} A(x, y)+t\left[\lambda_{2} B(x, y)+\lambda_{3} C x+\lambda_{4} D y\right] \\
= & \eta(t, x, y) \lambda_{1} A(x, y)+(\varphi(t)-\eta(t, x, y)) \lambda_{1} A(x, y) \\
& +t\left[\lambda_{2} B(x, y)+\lambda_{3} C x+\lambda_{4} D y\right] \\
\geq & \eta(t, x, y) \lambda_{1} A(x, y)+(\varphi(t)-\eta(t, x, y)) \frac{\eta(t, x, y)-t}{\varphi(t)-\eta(t, x, y)} \\
& \times\left[\lambda_{2} B(x, y)+\lambda_{3} C x+\lambda_{4} D y\right] \\
& +t\left[\lambda_{2} B(x, y)+\lambda_{3} C x+\lambda_{4} D y\right] \\
= & \eta(t, x, y)\left[\lambda_{1} A(x, y)+\lambda_{2} B(x, y)+\lambda_{3} C x+\lambda_{4} D y\right] \\
= & \eta(t, x, y) T(x, y), \quad \forall t \in(0,1), x, y \in P_{h} .
\end{aligned}
$$

Therefore, there exists $\eta(t, x, y) \in(t, 1]$ such that

$$
T\left(t x, t^{-1} y\right) \geq \eta(t, x, y) T(x, y), \quad \forall t \in(0,1), x, y \in P_{h} .
$$

Thirdly, we prove conclusion (C2). Owing to $T(h, h) \in P_{h}$, we have $t_{0} h \leq T(h, h) \leq t_{0}^{-1} h$, where $t_{0} \in(0,1)$ is a small constant we choose. As a result of $\eta(t, x, y) \in(t, 1]$, we have $1<\frac{\eta\left(t_{0}, x, y\right)}{t_{0}} \leq \frac{1}{t_{0}}$. By the Archimedes principle, we can take a positive integer $k$ such that $\left(\frac{\eta\left(t_{0}, x, y\right)}{t_{0}}\right)^{k} \geq \frac{1}{t_{0}}$. This inequality can be rewritten as

$$
\eta\left(t_{0}, x, y\right) \geq\left(\frac{1}{t_{0}}\right)^{\frac{1}{k}} \cdot t_{0}, \quad \frac{1}{\eta\left(t_{0}, x, y\right)} \leq \frac{1}{t_{0}} \cdot t_{0}^{\frac{1}{k}}
$$

Set $u_{0}=t_{0}^{k} h, v_{0}=t_{0}^{-k} h$. We can get $u_{0}, v_{0} \in P_{h}, u_{0}=t_{0}^{2 k} v_{0}<v_{0}$. Consequently, there exists $r \in$ $\left(0, t^{2 k}\right]$ such that $r \in(0,1), u_{0} \geq r v_{0}$. In addition, thanks to the mixed monotone properties of $A, B$, the increasing properties of $C$, and the decreasing properties of $D, T$ is a mixed monotone operator, and then $T\left(u_{0}, v_{0}\right) \leq T\left(v_{0}, u_{0}\right)$. From $(7)$, there is

$$
\begin{aligned}
T\left(u_{0}, v_{0}\right) & =T\left(t_{0}^{k} h, t_{0}^{-k} h\right) \\
& =T\left(t_{0} \cdot t_{0}^{k-1} h, t_{0}^{-1} \cdot t_{0}^{-k+1} h\right) \\
& \geq \eta\left(t_{0}, t_{0}^{k-1} h, t_{0}^{-k+1} h\right) T\left(t_{0}^{k-1} h, t_{0}^{-k+1} h\right) \\
& =\eta\left(t_{0}, t_{0}^{k-1} h, t_{0}^{-k+1} h\right) T\left(t_{0} \cdot t_{0}^{k-2} h, t_{0}^{-1} \cdot t_{0}^{-k+2} h\right) \\
& \geq \eta\left(t_{0}, t_{0}^{k-1} h, t_{0}^{-k+1} h\right) \cdot \eta\left(t_{0}, t_{0}^{k-2} h, t_{0}^{-k+2} h\right) T\left(t_{0}^{k-2} h, t_{0}^{-k+2} h\right) \geq \cdots \\
& \geq\left(\left(\frac{1}{t_{0}}\right)^{\frac{1}{k}} \cdot t_{0}\right)^{k} T(h, h) \geq \frac{1}{t_{0}} \cdot t_{0}^{k} t_{0} h=t_{0}^{k} h=u_{0} .
\end{aligned}
$$


By (7), we get $T\left(t^{-1} x, t y\right) \leq \eta\left(t, t^{-1} x, t y\right)^{-1} T(x, y), \forall t \in(0,1), x, y \in P_{h}$. Hence,

$$
\begin{aligned}
T\left(v_{0}, u_{0}\right) & =T\left(t_{0}^{-k} h, t_{0}^{k} h\right) \\
& =T\left(t_{0}^{-1} \cdot t_{0}^{-k+1} h, t_{0} \cdot t_{0}^{k-1} h\right) \\
& \leq \eta\left(t_{0}, t_{0}^{-k} h, t_{0}^{k} h\right)^{-1} T\left(t_{0}^{-k+1} h, t_{0}^{k-1} h\right) \\
& =\eta\left(t_{0}, t_{0}^{-k} h, t_{0}^{k} h\right)^{-1} T\left(t_{0}^{-1} \cdot t_{0}^{-k+2} h, t_{0} \cdot t_{0}^{k-2} h\right) \\
& \leq \eta\left(t_{0}, t_{0}^{-k} h, t_{0}^{k} h\right)^{-1} \cdot \eta\left(t_{0}, t_{0}^{-k+1} h, t_{0}^{k-1} h\right)^{-1} T\left(t_{0}^{-k+2} h, t_{0}^{k-2} h\right) \leq \cdots \\
& \leq\left(\frac{1}{t_{0}} \cdot t_{0}^{\frac{1}{k}}\right)^{k} T(h, h) \leq \frac{1}{t_{0}^{k}} \cdot t_{0} t_{0}^{-1} h=\frac{1}{t_{0}^{k}} h=v_{0} .
\end{aligned}
$$

Therefore, there is $u_{0} \leq T\left(u_{0}, v_{0}\right) \leq T\left(v_{0}, u_{0}\right) \leq v_{0}$. That is $(C 2)$.

Fourthly, we show that the operator equation $T(x, x)=x$ has a unique solution $x^{*} \in P_{h}$. For $u_{0}, v_{0}$, construct successively the sequences as follows:

$$
u_{n}=T\left(u_{n-1}, v_{n-1}\right), \quad v_{n}=T\left(v_{n-1}, u_{n-1}\right), \quad n=1,2, \ldots, n
$$

Thus, by (C2), we have $u_{1} \leq v_{1}$. And then due to the mixed monotone properties of operator $T$, there are $u_{n} \leq v_{n}, n=1,2,3, \ldots$, and

$$
u_{0} \leq u_{1} \leq \cdots \leq u_{n} \leq \cdots \leq v_{n} \leq \cdots \leq v_{1} \leq v_{0}
$$

Considering $x_{0} \geq r y_{0}$ and (8), there is $x_{n} \geq x_{0} \geq r y_{0} \geq r y_{n}(n=1,2,3, \ldots)$. Put

$$
t_{n}=\sup \left\{t>0 \mid x_{n} \geq t y_{n}\right\}, \quad n=1,2,3, \ldots
$$

It is clear that $x_{n} \geq t_{n} y_{n}$. Then from (8), there is $x_{n+1} \geq x_{n} \geq t_{n} y_{n} \geq t_{n} y_{n+1}, n=1,2,3, \ldots$ Hence, $t_{n+1} \geq t_{n}$, i.e., $\left\{t_{n}\right\}$ is increasing about $n$, and $\left\{t_{n}\right\} \subset(0,1]$. Assume $\lim _{n \rightarrow \infty} t_{n} \rightarrow t^{*}$, so $t^{*}=1$. Otherwise, $t \in(0,1)$. From (7) and $t_{n} \leq t^{*}$,

$$
\begin{aligned}
x_{n+1} & =T\left(x_{n}, y_{n}\right) \geq T\left(t_{n} y_{n}, t_{n}^{-1} x_{n}\right)=T\left(\frac{t_{n}}{t^{*}} t^{*} y_{n}, \frac{t^{*}}{t_{n}} \frac{1}{t^{*}} x_{n}\right) \\
& \geq \frac{t_{n}}{t^{*}} T\left(t^{*} y_{n}, \frac{1}{t^{*}} x_{n}\right) \geq \frac{t_{n}}{t^{*}} \eta\left(t^{*}, y_{n}, x_{n}\right) T\left(y_{n}, x_{n}\right)=\frac{t_{n}}{t^{*}} \eta\left(t^{*}, y_{n}, x_{n}\right) y_{n+1},
\end{aligned}
$$

combining with the definition of $t_{n}$, there is $t_{n+1} \geq \frac{t_{n}}{t^{*}} \eta\left(t^{*}, y_{n}, x_{n}\right)$. Then $\eta\left(t^{*}, y_{n}, x_{n}\right) \leq \frac{t^{*}}{t_{n}} t_{n+1}$. Owing to $t^{*}<\frac{\delta t^{*}+\varphi\left(t^{*}\right)}{\delta+1}<\eta\left(t^{*}, y_{n}, x_{n}\right) \leq \frac{t^{*}}{t_{n}} t_{n+1}, n=1,2,3, \ldots$, and $\lim _{n \rightarrow \infty} \frac{t^{*}}{t_{n}} t_{n+1}=t^{*}$, we know that

$$
t^{*}<\frac{\delta t^{*}+\varphi\left(t^{*}\right)}{\delta+1} \leq t^{*}
$$

which is a contradiction. Thus, $\lim _{n \rightarrow \infty} t_{n}=1$. For any natural number $p$, there is

$$
\begin{aligned}
& \theta \leq x_{n+p}-x_{n} \leq y_{n}-x_{n} \leq y_{n}-t_{n} y_{n}=\left(1-t_{n}\right) v_{n} \leq\left(1-t_{n}\right) v_{0}, \\
& \theta \leq y_{n}-y_{n+p} \leq y_{n}-x_{n} \leq\left(1-t_{n}\right) v_{0} .
\end{aligned}
$$


By the normality of cone $P$, there is

$$
\begin{aligned}
& \left\|x_{n+p}-x_{n}\right\| \leq N\left(1-t_{n}\right)\left\|v_{0}\right\| \rightarrow 0 \\
& \left\|y_{n}-y_{n+p}\right\| \leq N\left(1-t_{n}\right)\left\|v_{0}\right\| \rightarrow 0 \quad(n, p=1,2,3, \ldots),
\end{aligned}
$$

where $N$ is the normality constant. This shows that $\left\{x_{n}\right\}$ and $\left\{y_{n}\right\}$ are Cauchy sequences. Because of the complete continuity of $A$, there are $x_{n} \rightarrow x^{*}, y_{n} \rightarrow y^{*}$, when $n \rightarrow \infty$. And by (8), for $\forall x^{*}, y^{*} \in P_{h}$, we obtain that $x_{n} \leq x^{*} \leq y^{*} \leq y_{n}, \theta \leq y^{*}-x^{*} \leq y_{n}-x_{n} \leq\left(1-t_{n}\right) v_{0}$. Since cone $P$ is normal, we have

$$
\left\|y^{*}-x^{*}\right\| \leq N\left(1-t_{n}\right)\left\|v_{0}\right\| \rightarrow 0 \quad(n \rightarrow \infty),
$$

hence, $y^{*}=x^{*}$. Set $z^{*}:=y^{*}=x^{*}$, there is

$$
x_{n+1}=T\left(x_{n}, y_{n}\right) \leq T\left(z^{*}, z^{*}\right) \leq T\left(y_{n}, x_{n}\right)=y_{n+1},
$$

when $n \rightarrow \infty, z^{*}=T\left(z^{*}, z^{*}\right)$, i.e., $z^{*}$ is a fixed point of $T$ in $P_{h}$.

Next, we show that $z^{*}$ is the unique fixed point of $T$. Assume that $\bar{z}$ is another fixed point of $T$. Thanks to $z^{*}, \bar{z} \in P_{h}$, there exist positive numbers $a_{1}, a_{2}, b_{1}, b_{2}$ such that

$$
a_{1} h \leq z^{*} \leq a_{2} h, \quad b_{1} h \leq \bar{z} \leq b_{2} h,
$$

then $\bar{z} \leq b_{2} h=\frac{b_{2}}{a_{1}} a_{1} h \leq \frac{b_{2}}{a_{1}} z^{*}, \bar{z} \geq b_{1} h=\frac{b_{1}}{a_{2}} a_{2} h \geq \frac{b_{1}}{a_{2}} z^{*}$. We put

$$
e_{1}=\sup \left\{t>0, t z^{*} \leq \bar{z} \leq t^{-1} z^{*}\right\}
$$

Consequently, $0<e_{1} \leq 1, e_{1} z^{*} \leq \bar{z} \leq e_{1}^{-1} z^{*}$. Then $e_{1}=1$. Otherwise, $0<e_{1}<1$. There is

$$
\bar{z}=T(\bar{z}, \bar{z}) \geq T\left(e_{1} z^{*}, e_{1}^{-1} z^{*}\right) \geq \eta\left(e_{1}, z^{*}, z^{*}\right) T\left(z^{*}, z^{*}\right)=\eta\left(e_{1}, z^{*}, z^{*}\right) z^{*}
$$

Hence, $\eta\left(e_{1}, z^{*}, z^{*}\right) \geq e_{1}$, which contradicts the definition of $e_{1}$. So, $e_{1}=1$. Therefore, $z^{*}=\bar{z}$, i.e., $T$ has a unique fixed point $x^{*}$ in $P_{h}$.

Eventually, we show that conclusion $(C 4)$ holds. For any initial values $x_{0}, y_{0} \in P_{h}$, we construct the iterative sequences:

$$
x_{n}=T\left(x_{n-1}, y_{n-1}\right), \quad y_{n}=T\left(y_{n-1}, x_{n-1}\right), \quad n=1,2,3, \ldots
$$

Due to $x_{0}, y_{0} \in P_{h}$, we have $e_{2} h \leq x_{0} \leq e_{2}^{-1} h, e_{3} h \leq y_{0} \leq e_{3}^{-1} h$, where $e_{2}, e_{3} \in(0,1)$ are two small numbers. Put $e_{*}=\min \left\{e_{2}, e_{3}\right\}$, we deduce that $e_{*} \in(0,1)$ and $e_{*} h \leq x_{0}, y_{0} \leq e_{*}^{-1} h$. Then, by $e_{*}<\eta\left(e_{*}, x, y\right) \leq 1$, there is $1<\frac{\eta\left(e_{*}, x, y\right)}{e_{*}} \leq \frac{1}{e_{*}}$. By the Archimedes principle, there exists a sufficiently large positive integer $m$ such that

$$
\frac{\eta\left(e_{*}, x, y\right)}{e_{*}} \geq\left(\frac{1}{e_{*}}\right)^{\frac{1}{m}}
$$

Set $\bar{u}_{0}=e_{*}^{m} h, \bar{v}_{0}=\frac{1}{e_{*}^{m}} h$. Obviously, $\bar{u}_{0}, \bar{v}_{0} \in P_{h}$, and $\bar{u}_{0}<x_{0}, y_{0}<\bar{v}_{0}$. Put

$$
\bar{u}_{n}=T\left(\bar{u}_{n-1}, \bar{v}_{n-1}\right), \quad \bar{v}_{n}=T\left(\bar{v}_{n-1}, \bar{u}_{n-1}\right), \quad n=1,2,3, \ldots
$$


Similarly, there exists $y^{*} \in P_{h}$ such that $T\left(y^{*}, y^{*}\right)=y^{*}, \lim _{n \rightarrow \infty} \bar{u}_{n}=\lim _{n \rightarrow \infty} \bar{v}_{n}=y^{*}$. Because $T$ has the unique fixed points in $P_{h}$, we get $x^{*}=y^{*}$. And by induction, $\bar{u}_{n} \leq x_{n}, y_{n} \leq$ $\bar{v}_{n}, n=1,2,3, \ldots$. Thanks to cone $P$ being normal, there is $\lim _{n \rightarrow \infty} x_{n}=\lim _{n \rightarrow \infty} y_{n}=x^{*}$, i.e., conclusion (C4). The proof is complete.

Corollary 3.1 There are four operators $A, B, C, D$, where $A, B: P \times P \rightarrow P$ are two mixed monotone operators, $C: P \rightarrow P$ is an increasing homogeneous operator, and $D: P \rightarrow P$ is a decreasing operator. Then, if $\left(A_{2}\right)-\left(A_{5}\right)$ of Theorem 3.1 are satisfied and meet the following:

$\left(A_{6}\right)$ There exists $\gamma \in(0,1)$ such that

$$
A\left(\lambda x, \lambda^{-1} y\right) \geq \lambda^{\gamma} A(x, y), \quad D\left(\lambda^{-1} y\right) \geq \lambda D y, \quad \forall \lambda \in(0,1), x, y \in P
$$

then we can get conclusions (C1)-(C4) of Theorem 3.1.

Corollary 3.2 There are four operators $A, B, C, D$, where $A, B: P_{h} \times P_{h} \rightarrow P_{h}$ are two mixed monotone operators, $C: P_{h} \rightarrow P_{h}$ is an increasing sub-homogeneous operator, and $D: P_{h} \rightarrow P_{h}$ is a decreasing operator. Then, if $\left(A_{3}\right),\left(A_{5}\right)$ of Theorem 3.1 are satisfied and have the following:

$\left(A_{7}\right)$ There exists $\varphi(\lambda) \in(\lambda, 1]$ such that

$$
A\left(\lambda x, \lambda^{-1} y\right) \geq \varphi(\lambda) A(x, y), \quad D\left(\lambda^{-1} y\right) \geq \lambda D y, \quad \forall x, y \in P_{h}
$$

$\left(A_{8}\right)$ For any fixed $y \in P_{h}, B(\cdot, y): P_{h} \rightarrow P_{h}$ is concave; for any $x \in P_{h}, B(x, \cdot): P_{h} \rightarrow P_{h}$ is convex; then we can get conclusions $(C 2)-(C 4)$ of Theorem 3.1.

Corollary 3.3 There are four operators $A, B, C, D$, where $A, B: P_{h} \times P_{h} \rightarrow P_{h}$ are two mixed monotone operators, $C: P_{h} \rightarrow P_{h}$ is an increasing homogeneous operator, and $D$ : $P_{h} \rightarrow P_{h}$ is a decreasing operator. Then, if $\left(A_{3}\right),\left(A_{5}\right),\left(A_{7}\right),\left(A_{8}\right)$ are satisfied, then we can get conclusions (C2)-(C4) of Theorem 3.1.

Theorem 3.2 There are four operators $A, B, C, D$, where $A, B: P \times P \rightarrow P$ are two mixed monotone operators, $C: P \rightarrow P$ is an increasing operator, and $D: P \rightarrow P$ is a decreasing operator. Then, if conditions $\left(A_{2}\right)-\left(A_{4}\right)$ of Theorem 3.1 and the following conditions are satisfied:

$\left(A_{9}\right)$ There exists $\varphi(\lambda) \in(\lambda, 1]$ such that

$$
\begin{aligned}
& A\left(\lambda x, \lambda^{-1} y\right) \geq \lambda A(x, y), \quad C(\lambda x) \geq \varphi(\lambda) C x, \\
& D\left(\lambda^{-1} y\right) \geq \lambda D y, \quad \forall \lambda \in(0,1), x, y \in P
\end{aligned}
$$

$\left(A_{10}\right)$ There exists $\delta>0$ such that $\lambda_{1} A(x, y)+\lambda_{2} B(x, y)+\lambda_{4} D y \leq \delta \lambda_{3} C x, \forall x, y \in P_{h}$; then we can get conclusions (C1)-(C4) of Theorem 3.1.

Proof Define the operator $T=\lambda_{1} A+\lambda_{2} B+\lambda_{3} C+\lambda_{4} D$ by

$$
T(x, y)=\lambda_{1} A(x, y)+\lambda_{2} B(x, y)+\lambda_{3} C x+\lambda_{4} D y, \quad \forall x, y \in P .
$$


Firstly, we show that $T: P_{h} \times P_{h} \rightarrow P_{h}$. According to Theorem 3.1, there is $B: P_{h} \times P_{h} \rightarrow$ $P_{h}, D: P_{h} \rightarrow P_{h}$. Thus, we only prove that $A: P_{h} \times P_{h} \rightarrow P_{h}, C: P_{h} \rightarrow P_{h}$. Since (9), we obtain

$$
A\left(\lambda^{-1} x, \lambda y\right) \leq \lambda^{-1} A(x, y), \quad C\left(\lambda^{-1} x\right) \leq \varphi(\lambda)^{-1} C x, \quad \forall \lambda \in(0,1), x, y \in P .
$$

By $A(h, h), C h \in P_{h},(3)-(4),(6)$ hold. By deduction, for any $x, y \in P_{h}$, we can get

$$
\begin{aligned}
& A(x, y) \leq A\left(c_{1}^{-1} h, c_{2} h\right) \leq A\left(c^{-1} h, c h\right) \leq c^{-1} A(h, h) \leq c^{-1} \overline{v_{1}} h, \\
& A(x, y) \geq A\left(c_{1} h, c_{2}^{-1} h\right) \geq A\left(c h, c^{-1} h\right) \geq c A(h, h) \geq c \overline{u_{1}} h, \\
& C x \leq C\left(c_{1}^{-1} h\right) \leq C\left(c^{-1} h\right) \leq \varphi(c)^{-1} C h \leq \varphi(\lambda)^{-1} \overline{v_{3}} h, \\
& C x \geq C\left(c_{1} h\right) \geq C(c h) \geq \varphi(\lambda) C h \geq \varphi(\lambda) \overline{u_{3}} h,
\end{aligned}
$$

where $c=\min \left\{c_{1}, c_{2}\right\}$. It shows that $A: P_{h} \times P_{h} \rightarrow P_{h}, C: P_{h} \rightarrow P_{h}$. With $B: P_{h} \times P_{h} \rightarrow P_{h}$, $D: P_{h} \rightarrow P_{h}, T: P_{h} \times P_{h} \rightarrow P_{h}$ holds. Clearly, $T(h, h) \in P_{h}$.

Secondly, we prove that there exists $\eta(t, x, y) \in(t, 1]$ such that

$$
T\left(t x, t^{-1} y\right) \geq \eta(t, x, y) T(x, y), \quad \forall t \in(0,1), x, y \in P_{h} .
$$

By $A, B: P_{h} \times P_{h} \rightarrow P_{h}, C, D: P_{h} \rightarrow P_{h}$, we get $\lambda_{1} A(x, y)+\lambda_{2} B(x, y)+\lambda_{4} D y \sim \lambda_{3} C x$. Set

$$
J^{*}(x, y)=K\left(\frac{\lambda_{1} A(x, y)+\lambda_{2} B(x, y)+\lambda_{4} D y}{\lambda_{3} C x}\right) .
$$

Since $\left(A_{10}\right), J^{*}(x, y) \leq \delta$. Considering a similar function $h^{*}(s)=\frac{\varphi(t)+J(x, y) t}{\left.J^{*}(x, y)+1\right) s}$ of Theorem 3.1, $J^{*}(x, y)=\frac{\varphi(t)-\eta(t, x, y)}{\eta(t, x, y)-t}$ holds by Theorem 3.1. Thus, there is

$$
\lambda_{1} A(x, y)+\lambda_{2} B(x, y)+\lambda_{4} D y \leq \frac{\varphi(t)-\eta(t, x, y)}{\eta(t, x, y)-t} \lambda_{3} C x, \quad \forall t \in(0,1), x, y \in P_{h} .
$$

Combining with (5) and (9), for $\forall t \in(0,1), x, y \in P_{h}$, we obtain

$$
\begin{aligned}
T\left(t x, t^{-1} y\right)= & \lambda_{1} A\left(t x, t^{-1} y\right)+\lambda_{2} B\left(t x, t^{-1} y\right)+\lambda_{3} C(t x)+\lambda_{4} D\left(t^{-1} y\right) \\
\geq & t \lambda_{1} A(x, y)+t \lambda_{2} B(x, y)+\varphi(t) \lambda_{3} C x+t \lambda_{4} D y \\
= & \eta(t, x, y) \lambda_{3} C x+(\varphi(t)-\eta(t, x, y)) \lambda_{3} C x \\
& +t\left[\lambda_{1} A(x, y)+\lambda_{2} B(x, y)+\lambda_{4} D y\right] \\
= & \eta(t, x, y)\left[\lambda_{1} A(x, y)+\lambda_{2} B(x, y)+\lambda_{3} C x+\lambda_{4} D y\right] \\
= & \eta(t, x, y) T(x, y) .
\end{aligned}
$$

The next steps are the same as those of Theorem 3.1, then we can get conclusions (C1)(C4) of Theorem 3.1. 
Corollary 3.4 There are four operators $A, B, C, D$, where $A, B: P \times P \rightarrow P$ are two mixed monotone operators, $C: P \rightarrow P$ is an increasing operator, and $D: P \rightarrow P$ is a decreasing operator. Then, if conditions $\left(A_{2}\right)-\left(A_{4}\right)$ and $\left(A_{10}\right)$ of Theorem 3.1 and the following conditions are satisfied:

$\left(A_{11}\right)$ There exists $\gamma \in(0,1)$ such that

$$
\begin{aligned}
& A\left(\lambda x, \lambda^{-1} y\right) \geq \lambda A(x, y), \quad C(\lambda x) \geq \lambda^{\gamma} C x, \\
& D\left(\lambda^{-1} y\right) \geq \lambda D y, \quad \forall \lambda \in(0,1), x, y \in P
\end{aligned}
$$

then we can get conclusions $(C 1)-(C 4)$ of Theorem 3.1.

Corollary 3.5 There are four operators $A, B, C, D$, where $A, B: P_{h} \times P_{h} \rightarrow P_{h}$ are two mixed monotone operators, $C: P_{h} \rightarrow P_{h}$ is an increasing sub-homogeneous operator, and $D: P_{h} \rightarrow P_{h}$ is a decreasing operator. Then, if conditions $\left(A_{3}\right),\left(A_{8}\right),\left(A_{10}\right)$ and the following conditions are satisfied:

$\left(A_{12}\right)$ There exists $\gamma \in(0,1)$ such that

$$
\begin{aligned}
& A\left(\lambda x, \lambda^{-1} y\right) \geq \lambda A(x, y), \quad C(\lambda x) \geq \varphi(\lambda) C x, \\
& D\left(\lambda^{-1} y\right) \geq \lambda D y, \quad \forall x, y \in P_{h} ;
\end{aligned}
$$

then we can get conclusions (C2)-(C4) of Theorem 3.1.

\section{Application}

Nonlinear boundary value problems have attracted much attention for their applications in a variety of different areas such as mathematics, physics, biology, and so on. Thereafter there are a lot of interesting and important results, which include the uniqueness, existence, and multiplicity of positive solutions for the differential equation with the twopoint, three-point, infinite-point boundary value problems or some integral boundary problems, etc. In the following, we study a class of integro-differential boundary problems for fractional differential equations:

$$
\left\{\begin{array}{l}
D_{0^{+}}^{\alpha} x(t)+\lambda_{1} f(t, x(t), x(t))+\lambda_{2} g(t, x(t), x(t))+\lambda_{3} \phi(t, x(t))+\lambda_{4} \psi(t, x(t))=0, \\
x^{(i)}(0)=0, \quad i=0,1, \ldots, n-2, \\
D_{0^{+}}^{\beta} x(1)=\int_{0}^{\eta} x(s) \mathrm{d} s,
\end{array}\right.
$$

where $D_{0^{+}}^{\alpha}, D_{0^{+}}^{\beta}$ is Riemann-Liouville fractional derivative, $0<\beta<n-1<\alpha \leq n(n>1)$, $\alpha-\beta-1>0, t \in[0,1], \eta \in(0,1]$.

Definition 4.1 ([8]) Suppose that $h \in C[0,1], \alpha>0$. Then the Riemann-Liouville fractional derivative of $\alpha$ order is defined to be

$$
D_{0^{+}}^{\alpha} h(t)=\frac{1}{\Gamma(n-\alpha)} \frac{\mathrm{d}^{\mathrm{n}}}{\mathrm{d} t^{n}} \int_{0}^{t}(t-s)^{n-\alpha-1} h(s) \mathrm{d} s .
$$

The Riemann-Liouville fractional integral of $\alpha$ order is defined to be

$$
I_{0^{+}}^{\alpha} h(t)=\frac{1}{\Gamma(\alpha)} \int_{0}^{t}(t-s)^{\alpha-1} h(s) \mathrm{d} s
$$


where $n=[\alpha]+1,[\alpha]$ denotes the integer part of number $[\alpha]$, provided that the right-hand side is pointwise defined on $(0,1)$.

Lemma 4.1 Let $h \in C[0,1]$, the function $x$ is the solution of the following fractional differential equation:

$$
\left\{\begin{array}{l}
D_{0^{+}}^{\alpha} x(t)+h(t)=0, \quad n-1<\alpha \leq n \\
x^{(i)}(0)=0, \quad 0 \leq i \leq n-2, \\
D_{0^{+}}^{\beta} x(1)=\int_{0}^{\eta} x(s) \mathrm{d} s
\end{array}\right.
$$

if and only if $x$ satisfies

$$
x(t)=\int_{0}^{1} G(t, s) h(s) \mathrm{d} s,
$$

where

$$
G(t, s)=\left\{\begin{array}{l}
\frac{\Gamma(\alpha+1) t^{\alpha-1}(1-s)^{\alpha-\beta-1}-A(t-s)^{\alpha-1}-\Gamma(\alpha-\beta) t^{\alpha-1}(\eta-s)^{\alpha}}{A \Gamma(\alpha)}, \quad 0 \leq s \leq t \leq 1, s \leq \eta \\
\frac{\Gamma(\alpha+1) t^{\alpha-1}(1-s)^{\alpha-\beta-1}-A(t-s)^{\alpha-1}}{A \Gamma(\alpha)}, \quad 0 \leq \eta \leq s \leq t \leq 1 \\
\frac{\Gamma(\alpha+1) t^{\alpha-1}(1-s)^{\alpha-\beta-1}-\Gamma(\alpha-\beta) t^{\alpha-1}(\eta-s)^{\alpha}}{A \Gamma(\alpha)}, \quad 0 \leq t \leq s \leq \eta \leq 1 \\
\frac{\Gamma(\alpha+1) t^{\alpha-1}(1-s)^{\alpha-\beta-1}}{A \Gamma(\alpha)}, \quad 0 \leq t \leq s \leq 1, \eta \leq s
\end{array}\right.
$$

where $A=\Gamma(\alpha+1)-\Gamma(\alpha-\beta) \eta^{\alpha}$.

Proof Integrating on both sides of the first formula of (13), we can obtain

$$
\begin{aligned}
x(t) & =-I_{0^{+}}^{\alpha} h(t)+c_{1} t^{\alpha-1}+c_{2} t^{\alpha-2}+\cdots+c_{n} t^{\alpha-n} \\
& =-\int_{0}^{t} \frac{(t-s)^{\alpha-1}}{\Gamma(\alpha)} h(s) \mathrm{d} s+c_{1} t^{\alpha-1}+c_{2} t^{\alpha-2}+\cdots+c_{n} t^{\alpha-n} .
\end{aligned}
$$

By the boundary condition $x^{(i)}(0)=0(2 \leq i \leq n-2)$, we can easily get $c_{n}=c_{n-1}=\cdots=$ $c_{2}=0$. Then we can get the solution of equation (13) of the following form:

$$
x(t)=-I_{0^{+}}^{\alpha} h(t)+c_{1} t^{\alpha-1}=-\int_{0}^{t} \frac{(t-s)^{\alpha-1}}{\Gamma(\alpha)} h(s) \mathrm{d} s+c_{1} t^{\alpha-1} .
$$

By the equality $D_{0^{+}}^{\beta} t^{\alpha-1}=\frac{\Gamma(\alpha)}{\Gamma(\alpha-\beta)} t^{\alpha-\beta-1}$, we get

$$
D_{0^{+}}^{\beta} x(t)=-I_{0^{+}}^{\alpha-\beta} h(t)+D_{0^{+}}^{\beta} c_{1} t^{\alpha-\beta-1}=-\int_{0}^{t} \frac{(t-s)^{\alpha-\beta-1}}{\Gamma(\alpha-\beta)} h(s) \mathrm{d} s+c_{1} \frac{\Gamma(\alpha)}{\Gamma(\alpha-\beta)} t^{\alpha-\beta-1} .
$$

Then integrating formula (15) from 0 to $\eta$, there is

$$
\begin{aligned}
\int_{0}^{\eta} x(s) \mathrm{d} s & =-\int_{0}^{\eta} \int_{0}^{s} \frac{(s-\tau)^{\alpha-1}}{\Gamma(\alpha)} h(\tau) \mathrm{d} \tau \mathrm{d} s+\int_{0}^{\eta} c_{1} s^{\alpha-1} \mathrm{~d} s \\
& =-\int_{0}^{\eta} \frac{(\eta-s)^{\alpha}}{\Gamma(\alpha+1)} h(s) \mathrm{d} s+c_{1} \frac{\eta^{\alpha}}{\alpha} .
\end{aligned}
$$


By the condition $D_{0^{+}}^{\beta} x(1)=\int_{0}^{\eta} x(s) \mathrm{d} s$, we have

$$
c_{1}=\frac{1}{A}\left\{\int_{0}^{1} \alpha(1-s)^{\alpha-\beta-1} h(s) \mathrm{d} s-\int_{0}^{\eta} \frac{\Gamma(\alpha-\beta)}{\Gamma(\alpha)}(\eta-s)^{\alpha} h(s) \mathrm{d} s\right\},
$$

where $A$ is $\Gamma(\alpha+1)-\Gamma(\alpha-\beta) \eta^{\alpha}$. Substituting (16) in (15), we conclude

$$
\begin{aligned}
x(t)= & -\int_{0}^{t} \frac{(t-s)^{\alpha-1}}{\Gamma(\alpha)} h(s) \mathrm{d} s+c_{1} t^{\alpha-1} \\
= & -\int_{0}^{t} \frac{(t-s)^{\alpha-1}}{\Gamma(\alpha)} h(s) \mathrm{d} s+\frac{\alpha t^{\alpha-1}}{A} \int_{0}^{1}(1-s)^{\alpha-\beta-1} h(s) \mathrm{d} s \\
& -\frac{t^{\alpha-1} \Gamma(\alpha-\beta)}{A \Gamma(\alpha)} \int_{0}^{\eta}(\eta-s)^{\alpha} h(s) \mathrm{d} s \\
= & \int_{0}^{1} G(t, s) h(s) \mathrm{d} s,
\end{aligned}
$$

where $G(t, s)$ is defined as in (14).

Lemma 4.2 Let $G(t, s)$ be as given in (14). If $\Gamma(\alpha+1) \geq \Gamma(\alpha-\beta) \eta^{\alpha}$, then for any $t, s \in[0,1]$, we have

$$
\begin{aligned}
0 & \leq \frac{\Gamma(\alpha-\beta) \eta^{\alpha}\left[1-(1-s)^{\beta+1}\right](1-s)^{\alpha-\beta-1} t^{\alpha-1}}{A \Gamma(\alpha)} \\
& \leq G(t, s) \\
& \leq \frac{\Gamma(\alpha+1) t^{\alpha-1}(1-s)^{\alpha-\beta-1}}{A \Gamma(\alpha)} .
\end{aligned}
$$

Proof When $0 \leq s \leq t \leq 1, s \leq \eta$, by $s \leq \eta, \alpha \geq 0$, we observe that

$$
(1-s)^{\alpha}>\left(1-\frac{s}{\eta}\right)^{\alpha}
$$

By $s \leq t, \alpha-\beta-1 \geq 0$, we have

$$
t^{\alpha-1}(1-s)^{\alpha-\beta-1}>(t-s)^{\alpha-1} .
$$

Thus,

$$
\begin{aligned}
G(t, s) & =\frac{\Gamma(\alpha+1) t^{\alpha-1}(1-s)^{\alpha-\beta-1}-A(t-s)^{\alpha-1}-\Gamma(\alpha-\beta) t^{\alpha-1}(\eta-s)^{\alpha}}{A \Gamma(\alpha)} \\
& =\frac{\Gamma(\alpha+1) t^{\alpha-1}(1-s)^{\alpha-\beta-1}-\Gamma(\alpha-\beta) t^{\alpha-1} \eta^{\alpha}\left(1-\frac{s}{\eta}\right)^{\alpha}}{\left[\Gamma(\alpha+1)-\Gamma(\alpha-\beta) \eta^{\alpha}\right] \Gamma(\alpha)}-\frac{(t-s)^{\alpha-1}}{\Gamma(\alpha)} \\
& \geq \frac{\Gamma(\alpha+1) t^{\alpha-1}(1-s)^{\alpha-\beta-1}-\Gamma(\alpha-\beta) t^{\alpha-1} \eta^{\alpha}(1-s)^{\alpha}}{\left[\Gamma(\alpha+1)-\Gamma(\alpha-\beta) \eta^{\alpha}\right] \Gamma(\alpha)}-\frac{(t-s)^{\alpha-1}}{\Gamma(\alpha)} \\
& =\frac{\left[\Gamma(\alpha+1)-\Gamma(\alpha-\beta) \eta^{\alpha}(1-s)^{\beta+1}\right] t^{\alpha-1}(1-s)^{\alpha-\beta-1}}{\left[\Gamma(\alpha+1)-\Gamma(\alpha-\beta) \eta^{\alpha}\right] \Gamma(\alpha)}-\frac{(t-s)^{\alpha-1}}{\Gamma(\alpha)} \\
& =\frac{t^{\alpha-1}(1-s)^{\alpha-\beta-1}}{\Gamma(\alpha)}+\frac{\Gamma(\alpha-\beta) \eta^{\alpha}\left[1-(1-s)^{\beta+1}\right] t^{\alpha-1}(1-s)^{\alpha-\beta-1}}{\left[\Gamma(\alpha+1)-\Gamma(\alpha-\beta) \eta^{\alpha}\right] \Gamma(\alpha)}
\end{aligned}
$$




$$
\begin{aligned}
& -\frac{(t-s)^{\alpha-1}}{\Gamma(\alpha)} \\
= & \frac{t^{\alpha-1}(1-s)^{\alpha-\beta-1}-(t-s)^{\alpha-1}}{\Gamma(\alpha)}+\frac{\Gamma(\alpha-\beta) \eta^{\alpha}\left[1-(1-s)^{\beta+1}\right] t^{\alpha-1}(1-s)^{\alpha-\beta-1}}{\left[\Gamma(\alpha+1)-\Gamma(\alpha-\beta) \eta^{\alpha}\right] \Gamma(\alpha)} \\
= & \frac{t^{\alpha-1}(1-s)^{\alpha-\beta-1}-t^{\alpha-1}\left(1-\frac{s}{t}\right)^{\alpha-1}}{\Gamma(\alpha)} \\
& +\frac{\Gamma(\alpha-\beta) \eta^{\alpha}\left[1-(1-s)^{\beta+1}\right] t^{\alpha-1}(1-s)^{\alpha-\beta-1}}{\left[\Gamma(\alpha+1)-\Gamma(\alpha-\beta) \eta^{\alpha}\right] \Gamma(\alpha)} \\
\geq & \frac{\Gamma(\alpha-\beta) \eta^{\alpha}\left[1-(1-s)^{\beta+1}\right] t^{\alpha-1}(1-s)^{\alpha-\beta-1}}{\left[\Gamma(\alpha+1)-\Gamma(\alpha-\beta) \eta^{\alpha}\right] \Gamma(\alpha)} \\
= & \frac{\Gamma(\alpha-\beta) \eta^{\alpha}\left[1-(1-s)^{\beta+1}\right] t^{\alpha-1}(1-s)^{\alpha-\beta-1}}{A \Gamma(\alpha)} \geq 0,
\end{aligned}
$$

and

$$
\begin{aligned}
G(t, s) & =\frac{\Gamma(\alpha+1) t^{\alpha-1}(1-s)^{\alpha-\beta-1}-A(t-s)^{\alpha-1}-\Gamma(\alpha-\beta) t^{\alpha-1}(\eta-s)^{\alpha}}{A \Gamma(\alpha)} \\
& \leq \frac{\Gamma(\alpha+1) t^{\alpha-1}(1-s)^{\alpha-\beta-1}}{A \Gamma(\alpha)} .
\end{aligned}
$$

When $0 \leq \eta \leq s \leq t \leq 1$, from (18), there is

$$
\begin{aligned}
G(t, s) & =\frac{\Gamma(\alpha+1) t^{\alpha-1}(1-s)^{\alpha-\beta-1}-A(t-s)^{\alpha-1}}{A \Gamma(\alpha)} \\
& =\frac{\Gamma(\alpha+1) t^{\alpha-1}(1-s)^{\alpha-\beta-1}}{\left[\Gamma(\alpha+1)-\Gamma(\alpha-\beta) \eta^{\alpha}\right] \Gamma(\alpha)}-\frac{(t-s)^{\alpha-1}}{\Gamma(\alpha)} \\
& \geq \frac{\left[\Gamma(\alpha+1)-\Gamma(\alpha-\beta) \eta^{\alpha}(1-s)^{\beta+1}\right] t^{\alpha-1}(1-s)^{\alpha-\beta-1}}{\left[\Gamma(\alpha+1)-\Gamma(\alpha-\beta) \eta^{\alpha}\right] \Gamma(\alpha)}-\frac{(t-s)^{\alpha-1}}{\Gamma(\alpha)} \\
& =\frac{t^{\alpha-1}(1-s)^{\alpha-\beta-1}}{\Gamma(\alpha)}+\frac{\Gamma(\alpha-\beta) \eta^{\alpha}\left[1-(1-s)^{\beta+1}\right] t^{\alpha-1}(1-s)^{\alpha-\beta-1}}{\left[\Gamma(\alpha+1)-\Gamma(\alpha-\beta) \eta^{\alpha}\right] \Gamma(\alpha)}-\frac{(t-s)^{\alpha-1}}{\Gamma(\alpha)} \\
& =\frac{t^{\alpha-1}(1-s)^{\alpha-\beta-1}-(t-s)^{\alpha-1}}{\Gamma(\alpha)}+\frac{\Gamma(\alpha-\beta) \eta^{\alpha}\left[1-(1-s)^{\beta+1}\right] t^{\alpha-1}(1-s)^{\alpha-\beta-1}}{\left[\Gamma(\alpha+1)-\Gamma(\alpha-\beta) \eta^{\alpha}\right] \Gamma(\alpha)} \\
& \geq \frac{\Gamma(\alpha-\beta) \eta^{\alpha}\left[1-(1-s)^{\beta+1}\right] t^{\alpha-1}(1-s)^{\alpha-\beta-1}}{\left[\Gamma(\alpha+1)-\Gamma(\alpha-\beta) \eta^{\alpha}\right] \Gamma(\alpha)} \\
& =\frac{\Gamma(\alpha-\beta) \eta^{\alpha}\left[1-(1-s)^{\beta+1}\right] t^{\alpha-1}(1-s)^{\alpha-\beta-1}}{A \Gamma(\alpha)} \geq 0,
\end{aligned}
$$

and

$$
\begin{aligned}
G(t, s) & =\frac{\Gamma(\alpha+1) t^{\alpha-1}(1-s)^{\alpha-\beta-1}-A(t-s)^{\alpha-1}}{A \Gamma(\alpha)} \\
& \leq \frac{\Gamma(\alpha+1) t^{\alpha-1}(1-s)^{\alpha-\beta-1}}{A \Gamma(\alpha)} .
\end{aligned}
$$

When $0 \leq t \leq s \leq \eta \leq 1$,

$$
G(t, s)=\frac{\Gamma(\alpha+1) t^{\alpha-1}(1-s)^{\alpha-\beta-1}-\Gamma(\alpha-\beta) t^{\alpha-1}(\eta-s)^{\alpha}}{A \Gamma(\alpha)}
$$




$$
\begin{aligned}
& \geq \frac{\Gamma(\alpha+1) t^{\alpha-1}(1-s)^{\alpha-\beta-1}-\Gamma(\alpha-\beta) t^{\alpha-1} \eta^{\alpha}(1-s)^{\alpha}}{\left[\Gamma(\alpha+1)-\Gamma(\alpha-\beta) \eta^{\alpha}\right] \Gamma(\alpha)} \\
& =\frac{\left[\Gamma(\alpha+1)-\Gamma(\alpha-\beta) \eta^{\alpha}(1-s)^{\beta+1}\right] t^{\alpha-1}(1-s)^{\alpha-\beta-1}}{\left[\Gamma(\alpha+1)-\Gamma(\alpha-\beta) \eta^{\alpha}\right] \Gamma(\alpha)} \\
& =\frac{t^{\alpha-1}(1-s)^{\alpha-\beta-1}}{\Gamma(\alpha)}+\frac{\Gamma(\alpha-\beta) \eta^{\alpha}\left[1-(1-s)^{\beta+1}\right] t^{\alpha-1}(1-s)^{\alpha-\beta-1}}{\left[\Gamma(\alpha+1)-\Gamma(\alpha-\beta) \eta^{\alpha}\right] \Gamma(\alpha)} \\
& \geq \frac{\Gamma(\alpha-\beta) \eta^{\alpha}\left[1-(1-s)^{\beta+1}\right] t^{\alpha-1}(1-s)^{\alpha-\beta-1}}{A \Gamma(\alpha)} \geq 0
\end{aligned}
$$

and

$$
\begin{aligned}
G(t, s) & =\frac{\Gamma(\alpha+1) t^{\alpha-1}(1-s)^{\alpha-\beta-1}-\Gamma(\alpha-\beta) t^{\alpha-1}(\eta-s)^{\alpha}}{A \Gamma(\alpha)} \\
& \leq \frac{\Gamma(\alpha+1) t^{\alpha-1}(1-s)^{\alpha-\beta-1}}{A \Gamma(\alpha)} .
\end{aligned}
$$

When $0 \leq t \leq s \leq \eta \leq 1$,

$$
\begin{aligned}
G(t, s) & =\frac{\Gamma(\alpha+1) t^{\alpha-1}(1-s)^{\alpha-\beta-1}}{A \Gamma(\alpha)} \\
& \geq \frac{\left[\Gamma(\alpha+1)-\Gamma(\alpha-\beta) \eta^{\alpha}(1-s)^{\beta+1}\right] t^{\alpha-1}(1-s)^{\alpha-\beta-1}}{\left[\Gamma(\alpha+1)-\Gamma(\alpha-\beta) \eta^{\alpha}\right] \Gamma(\alpha)} \\
& \geq \frac{\Gamma(\alpha-\beta) \eta^{\alpha}\left[1-(1-s)^{\beta+1}\right] t^{\alpha-1}(1-s)^{\alpha-\beta-1}}{A \Gamma(\alpha)} \geq 0 .
\end{aligned}
$$

It is obvious that

$$
G(t, s) \leq \frac{\Gamma(\alpha+1) t^{\alpha-1}(1-s)^{\alpha-\beta-1}}{A \Gamma(\alpha)} .
$$

Then, for $\forall t, s \in[0,1]$, we obtain that $G(t, s)$ meets (17).

Theorem 4.1 Let $f, g:[0,1] \times[0,+\infty) \times[0,+\infty) \rightarrow[0,+\infty), \phi, \psi:[0,1] \times[0,+\infty) \rightarrow$ $[0,+\infty)$ be continuous with $f(t, 0,1), g(t, 0,1), \phi(t, 0), \psi(t, 1) \not \equiv 0$ and satisfy the following conditions:

$\left(N_{1}\right)$ For any fixed $t \in[0,1], y \in[0,+\infty), f(t, x, y), g(t, x, y), \phi(t, x)$ are increasing in $x \in$ $[0,+\infty)$; for any fixed $t \in[0,1], x \in[0,+\infty), f(t, x, y), g(t, x, y), \psi(t, y)$ are decreasing in $y \in[0,+\infty)$;

$\left(N_{2}\right)$ For $\forall \lambda \in(0,1), t \in[0,1], x, y \in[0,+\infty)$, there exists $\varphi(\lambda) \in(\lambda, 1]$ such that

$$
\begin{aligned}
& f\left(t, \lambda x, \lambda^{-1} y\right) \geq \varphi(\lambda) f(t, x, y), \quad \phi(t, \lambda x) \geq \lambda \phi(t, x), \\
& \psi\left(t, \lambda^{-1} y\right) \geq \lambda \psi(t, y)
\end{aligned}
$$

and for fixed $t \in[0,1], y \in[0,+\infty), g(t, \cdot, y)$ is concave; for fixed $t \in[0,1], x \in[0,+\infty)$, $g(t, x, \cdot)$ is convex;

$\left(N_{3}\right)$ Let $h(t)=t^{\alpha-1}$, there exists $c \geq 0$ such that $g(s, \theta, l h) \geq \operatorname{cg}(s, l h, \theta), l \geq 1$;

$\left(N_{4}\right)$ There exists a constant $\delta>0$ such that $\left[\lambda_{2} g(t, x, y)+\lambda_{3} \phi(t, x)+\lambda_{4} \psi(t, y)\right] \leq$ $\delta \lambda_{1} f(t, x, y), \forall t \in[0,1], x, y \in[0,+\infty)$. 
Then

(S1) There exist $u_{0}, v_{0} \in P_{h}, r \in(0,1)$ such that $r v_{0} \leq u_{0}<v_{0}$,

$$
\begin{aligned}
u_{0} \leq & \lambda_{1} \int_{0}^{1} G(t, s) f\left(s, u_{0}(s), v_{0}(s)\right) \mathrm{d} s+\lambda_{2} \int_{0}^{1} G(t, s) g\left(s, u_{0}(s), v_{0}(s)\right) \mathrm{d} s \\
& +\lambda_{3} \int_{0}^{1} G(t, s) \phi\left(s, u_{0}(s)\right) \mathrm{d} s+\lambda_{4} \int_{0}^{1} G(t, s) \psi\left(s, v_{0}(s)\right) \mathrm{d} s \\
\leq & \lambda_{1} \int_{0}^{1} G(t, s) f\left(s, v_{0}(s), u_{0}(s)\right) \mathrm{d} s+\lambda_{2} \int_{0}^{1} G(t, s) g\left(s, v_{0}(s), u_{0}(s)\right) \mathrm{d} s \\
& +\lambda_{3} \int_{0}^{1} G(t, s) \phi\left(s, v_{0}(s)\right) \mathrm{d} s+\lambda_{4} \int_{0}^{1} G(t, s) \psi\left(s, u_{0}(s)\right) \mathrm{d} s \leq v_{0} ;
\end{aligned}
$$

(S2) Equation (12) has a unique positive solution $x^{*}$ in $P_{h}$, which meets $\mu_{1} t^{\alpha-1} \leq x^{*} \leq$ $v_{1} t^{\alpha-1}, \mu_{1}, v_{1}>0$ are two constants;

(S3) For any initial values $x_{0}, y_{0} \in P_{h}$, we construct successively the iterative sequences

$$
\begin{aligned}
x_{n}(t)= & \lambda_{1} \int_{0}^{1} G(t, s) f\left(s, x_{n-1}(s), y_{n-1}(s)\right) \mathrm{d} s+\lambda_{2} \int_{0}^{1} G(t, s) g\left(s, x_{n-1}(s), y_{n-1}(s)\right) \mathrm{d} s \\
& +\lambda_{3} \int_{0}^{1} G(t, s) \phi\left(s, x_{n-1}(s)\right) \mathrm{d} s \\
& +\lambda_{4} \int_{0}^{1} G(t, s) \psi\left(s, y_{n-1}(s)\right) \mathrm{d} s, \quad n=1,2, \ldots, \\
y_{n}(t)= & \lambda_{1} \int_{0}^{1} G(t, s) f\left(s, y_{n-1}(s), x_{n-1}(s)\right) \mathrm{d} s+\lambda_{2} \int_{0}^{1} G(t, s) g\left(s, y_{n-1}(s), x_{n-1}(s)\right) \mathrm{d} s \\
& +\lambda_{1} \int_{0}^{1} G(t, s) \phi\left(s, y_{n-1}(s)\right) \mathrm{d} s \\
& +\lambda_{4} \int_{0}^{1} G(t, s) \phi\left(s, x_{n-1}(s)\right) \mathrm{d} s, \quad n=1,2, \ldots
\end{aligned}
$$

Here, $x_{n} \rightarrow x^{*}, y_{n} \rightarrow x^{*}$, when $n \rightarrow \infty$.

Proof Let $E=C[0,1]$ and $\|x\|=\sup _{0 \leq t \leq 1}|x(t)|$. It is obvious that $(E,\|\cdot\|)$ is a Banach space. Set $P=\{x \in E \mid x(t) \geq 0, t \in[0,1]\}$.

By Lemma 4.1, the unique solution of problem (12) has an integral formulation:

$$
x(t)=\int_{0}^{1} G(t, s)\left[\lambda_{1} f(s, x(s), x(s))+\lambda_{2} g(s, x(s), x(s))+\lambda_{3} \phi(s, x(s))+\lambda_{4} \psi(s, x(s))\right] \mathrm{d} s .
$$

Define four operators $A, B, C, D: P \times P \rightarrow E$ by

$$
\begin{aligned}
& A(x, y)(t)=\int_{0}^{1} G(t, s) f(s, x(s), y(s)) \mathrm{d} s, \\
& B(x, y)(t)=\int_{0}^{1} G(t, s) g(s, x(s), y(s)) \mathrm{d} s, \\
& C x(t)=\int_{0}^{1} G(t, s) \phi(s, x(s)) \mathrm{d} s,
\end{aligned}
$$




$$
D y(t)=\int_{0}^{1} G(t, s) \psi(s, y(s)) \mathrm{d} s .
$$

Therefore, $x$ is the solution of equation (12) if and only if $x$ is the solution of operator equation $x=\lambda_{1} A(x, x)+\lambda_{2} B(x, x)+\lambda_{3} C x+\lambda_{4} D x$.

Firstly, for $\forall x, y \in P$, in view of the definition of $f, g, \phi, \psi$ and Lemma 4.2, it is easy to obtain $A(x, y) \geq 0, B(x, y) \geq 0, C x \geq 0, D y \geq 0$, which shows that $A, B: P \times P \rightarrow P, C, D$ : $P \rightarrow P$. In addition, due to $\left(N_{1}\right), A, B$ are two monotone operators, $C$ is an increasing operator, and $D$ is a decreasing operator.

Secondly, we illustrate that $C$ is a sub-homogeneous operator and $A, D$ satisfy condition $\left(A_{1}\right)$ of Theorem 3.1. From $\left(N_{2}\right)$, we see that, for any $\lambda \in(0,1), x \in P$,

$$
\begin{aligned}
C(\lambda x) & =\int_{0}^{1} G(t, s) \phi(s, \lambda x(s)) \mathrm{d} s \\
& \geq \int_{0}^{1} G(t, s) \lambda \phi(s, x(s)) \mathrm{d} s \\
& =\lambda \int_{0}^{1} G(t, s) \phi(s, x(s)) \mathrm{d} s \\
& =\lambda C x,
\end{aligned}
$$

which shows that $C$ is a sub-homogeneous operator. Using $\left(N_{2}\right)$ again, $\forall \lambda \in(0,1), x, y \in P$, there exists $\varphi(\lambda) \in(\lambda, 1]$ such that

$$
\begin{aligned}
A\left(\lambda x, \lambda^{-1} y\right) & =\int_{0}^{1} G(t, s) f\left(s, \lambda x(s), \lambda^{-1} y(s)\right) \mathrm{d} s \\
& \geq \int_{0}^{1} G(t, s) \varphi(\lambda) f(s, x(s), y(s)) \mathrm{d} s \\
& =\varphi(\lambda) \int_{0}^{1} G(t, s) f(s, x(s), y(s)) \mathrm{d} s \\
& =\varphi(\lambda) A(x, y),
\end{aligned}
$$

and for any $\lambda \in(0,1), y \in P$,

$$
\begin{aligned}
D\left(\lambda^{-1} y\right) & =\int_{0}^{1} G(t, s) \psi\left(s, \lambda^{-1} y(s)\right) \mathrm{d} s \\
& \geq \int_{0}^{1} G(t, s) \lambda \psi(s, y(s)) \mathrm{d} s \\
& =\lambda \int_{0}^{1} G(t, s) \psi(s, y(s)) \mathrm{d} s \\
& =\lambda D y .
\end{aligned}
$$

Thus, operator $A, D$ satisfies $\left(A_{1}\right)$ of Theorem 3.1.

Thirdly, we verified condition $\left(A_{2}\right)$ of Theorem 3.1. From $\left(N_{2}\right)$, for fixed $t \in(0,1), y \in P$,

$$
\begin{aligned}
& B\left(\tau x_{1}+(1-\tau) x_{2}, y\right)(t) \\
& \quad=\int_{0}^{1} G(t, s) g\left(s, \tau x_{1}(s)+(1-\tau) x_{2}(s), y(s)\right) \mathrm{d} s
\end{aligned}
$$




$$
\begin{aligned}
& \geq \int_{0}^{1} G(t, s)\left[\tau g\left(s, x_{1}(s), y(s)\right)+(1-\tau) g\left(s, x_{2}(s), y(s)\right)\right] \mathrm{d} s \\
& =\tau \int_{0}^{1} G(t, s) g\left(s, x_{1}(s), y(s)\right) \mathrm{d} s+(1-\tau) \int_{0}^{1} G(t, s) g\left(s, x_{2}(s), y(s)\right) \mathrm{d} s \\
& =\tau B\left(x_{1}, y\right)+(1-\tau) B\left(x_{2}, y\right), \quad \forall \tau \in(0,1), x_{1}, x_{2} \in P ;
\end{aligned}
$$

for fixed $t \in(0,1), x \in P$,

$$
\begin{aligned}
B & \left(x, \tau y_{1}+(1-\tau) y_{2}\right)(t) \\
& =\int_{0}^{1} G(t, s) g\left(s, x(s), \tau y_{1}(s)+(1-\tau) y_{2}(s)\right) \mathrm{d} s \\
& \leq \int_{0}^{1} G(t, s)\left[\tau g\left(s, x(s), y_{1}(s)\right)+(1-\tau) g\left(s, x(s), y_{2}(s)\right)\right] \mathrm{d} s \\
& =\tau \int_{0}^{1} G(t, s) g\left(s, x(s), y_{1}(s)\right) \mathrm{d} s+(1-\tau) \int_{0}^{1} G(t, s) g\left(s, x(s), y_{2}(s)\right) \mathrm{d} s \\
& =\tau B\left(x, y_{1}\right)+(1-\tau) B\left(x, y_{2}\right), \quad \forall \tau \in(0,1), y_{1}, y_{2} \in P,
\end{aligned}
$$

which indicate that, for fixed $y \in P, B(\cdot, y)$ is concave; for fixed $x \in P, B(x, \cdot)$ is convex. That is condition $\left(A_{2}\right)$ of Theorem 3.1.

Fourthly, we check condition $\left(A_{3}\right)$ of Theorem 3.1. By $\left(N_{3}\right)$, there exists $\widetilde{c} \geq 0$ such that

$$
\begin{aligned}
B(\theta, \operatorname{lh})(t) & =\int_{0}^{1} G(t, s) g(s, \theta, \operatorname{lh}(s)) \mathrm{d} s \\
& \geq \widetilde{c} \int_{0}^{1} G(t, s) g(s, \operatorname{lh}(s), \theta) \mathrm{d} s \\
& =\widetilde{c} B(\operatorname{lh}, \theta)(t) .
\end{aligned}
$$

In the next step, we prove that $A(h, h), B(h, h), C h, D h \in P_{h}$. By Lemma 4.2, we have

$$
\begin{aligned}
A(h, h)(t) & =\int_{0}^{1} G(t, s) f\left(s, s^{\alpha-1}, s^{\alpha-1}\right) \mathrm{d} s \\
& \geq \frac{\Gamma(\alpha-\beta) \eta^{\alpha}}{A \Gamma(\alpha)} \int_{0}^{1}\left[1-(1-s)^{\beta+1}\right](1-s)^{\alpha-\beta-1} f\left(s, s^{\alpha-1}, s^{\alpha-1}\right) \mathrm{d} s \\
& \geq\left\{\frac{\Gamma(\alpha-\beta) \eta^{\alpha}}{A \Gamma(\alpha)} \int_{0}^{1}\left[1-(1-s)^{\beta+1}\right](1-s)^{\alpha-\beta-1} f(s, 0,1) \mathrm{d} s\right\} h(t),
\end{aligned}
$$

and

$$
\begin{aligned}
A(h, h)(t) & =\int_{0}^{1} G(t, s) f\left(s, s^{\alpha-1}, s^{\alpha-1}\right) \mathrm{d} s \\
& \leq \frac{\Gamma(\alpha+1) t^{\alpha-1}}{A \Gamma(\alpha)} \int_{0}^{1}(1-s)^{\alpha-\beta-1} f\left(s, s^{\alpha-1}, s^{\alpha-1}\right) \mathrm{d} s \\
& \leq\left\{\frac{\Gamma(\alpha+1)}{A \Gamma(\alpha)} \int_{0}^{1}(1-s)^{\alpha-\beta-1} f(s, 1,0) \mathrm{d} s\right\} h(t),
\end{aligned}
$$


where

$$
\begin{aligned}
& c_{1}=\frac{\Gamma(\alpha-\beta) \eta^{\alpha}}{A \Gamma(\alpha)} \int_{0}^{1}\left[1-(1-s)^{\beta+1}\right](1-s)^{\alpha-\beta-1} f(s, 0,1) \mathrm{d} s, \\
& c_{2}=\frac{\Gamma(\alpha+1)}{A \Gamma(\alpha)} \int_{0}^{1}(1-s)^{\alpha-\beta-1} f(s, 1,0) \mathrm{d} s .
\end{aligned}
$$

Thus, we can get $c_{1} h(t) \leq A(h, h)(t) \leq c_{2} h(t)$. Besides, due to $f(s, 1,0) \geq f(s, 0,1) \geq 0$ and $f(s, 0,1) \not \equiv 0$, we derive that

$$
\int_{0}^{1} f(s, 1,0) \mathrm{d} s \geq \int_{0}^{1} f(s, 0,1) \mathrm{d} s>0,
$$

thus, $A(h, h) \in P_{h}$. Similarly, there are

$$
\begin{aligned}
& \left\{\frac{\Gamma(\alpha-\beta) \eta^{\alpha}}{A \Gamma(\alpha)} \int_{0}^{1}\left[1-(1-s)^{\beta+1}\right](1-s)^{\alpha-\beta-1} g(s, 0,1) \mathrm{d} s\right\} h(t) \\
& \leq B(h, h)(t) \leq\left\{\frac{\Gamma(\alpha+1)}{A \Gamma(\alpha)} \int_{0}^{1}(1-s)^{\alpha-\beta-1} g(s, 1,0) \mathrm{d} s\right\} h(t), \\
& \left\{\frac{\Gamma(\alpha-\beta) \eta^{\alpha}}{A \Gamma(\alpha)} \int_{0}^{1}\left[1-(1-s)^{\beta+1}\right](1-s)^{\alpha-\beta-1} \phi(s, 0) \mathrm{d} s\right\} h(t) \\
& \leq C h(t) \leq\left\{\frac{\Gamma(\alpha+1)}{A \Gamma(\alpha)} \int_{0}^{1}(1-s)^{\alpha-\beta-1} \phi(s, 1) \mathrm{d} s\right\} h(t),
\end{aligned}
$$

and

$$
\begin{gathered}
\left\{\frac{\Gamma(\alpha-\beta) \eta^{\alpha}}{A \Gamma(\alpha)} \int_{0}^{1}\left[1-(1-s)^{\beta+1}\right](1-s)^{\alpha-\beta-1} \psi(s, 1) \mathrm{d} s\right\} h(t) \\
\leq D h(t) \leq\left\{\frac{\Gamma(\alpha+1)}{A \Gamma(\alpha)} \int_{0}^{1}(1-s)^{\alpha-\beta-1} \psi(s, 0) \mathrm{d} s\right\} h(t) .
\end{gathered}
$$

Then, by $g(s, 0,1), \phi(s, 0), \psi(s, 1) \not \equiv 0$, we can obtain $B(h, h), C h, D h \in P_{h}$.

Eventually, we test condition $\left(A_{5}\right)$ of Theorem 3.1. From $\left(N_{5}\right)$, for any $t \in[0,1], x, y \in P$,

$$
\begin{aligned}
& {\left[\lambda_{2} B(x, y)(t)+\lambda_{3} C x(t)+\lambda_{4} D y(t)\right]} \\
& \quad=\int_{0}^{1} G(t, s)\left[\lambda_{2} g(s, x(s), y(s))+\lambda_{3} \phi(s, x(s))+\lambda_{4} \psi(s, y(s))\right] \mathrm{d} s \\
& \quad \leq \delta \int_{0}^{1} G(t, s) \lambda_{1} f(s, x(s), y(s)) \mathrm{d} s \\
& \quad=\delta \lambda_{1} A(x, y)(t), \quad n=1,2, \ldots
\end{aligned}
$$

From the above six steps, we verified all the conditions of Theorem 3.1, thus the conclusions of Theorem 4.1 hold with Theorem 3.1.

Theorem 4.2 Let $f, g:[0,1] \times[0,+\infty) \times[0,+\infty) \rightarrow[0,+\infty), \phi:[0,1] \times[0,+\infty) \rightarrow$ $[0,+\infty)$, and $\psi:[0,1] \times[0,+\infty) \rightarrow[0,+\infty)$ are continuous with $f(t, 0,1), g(t, 0,1), \phi(t, 0)$, $\psi(t, 1) \not \equiv 0$. Then, if conditions $\left(N_{1}\right),\left(N_{4}\right)$ and the following conditions are satisfied: 
$\left(N_{5}\right)$ For $\forall \lambda \in(0,1), t \in[0,1], x, y \in[0,+\infty)$, there exists $\varphi(\lambda) \in(\lambda, 1]$ such that

$$
\begin{aligned}
& f\left(t, \lambda x, \lambda^{-1} y\right) \geq \lambda f(t, x, y), \quad \phi(t, \lambda x) \geq \varphi(\lambda) \phi(t, x) \\
& \psi\left(t, \lambda^{-1} y\right) \geq \lambda^{-1} \psi(t, y)
\end{aligned}
$$

and for fixed $t \in[0,1], y \in[0,+\infty), g(t, \cdot, y)$ is concave; for fixed $t \in[0,1], x \in[0,+\infty)$, $g(t, x, \cdot)$ is convex;

$\left(N_{6}\right)$ There exists a constant $\delta>0$ such that $\lambda_{1} f(t, x, y)+\lambda_{2} g(t, x, y)+\lambda_{4} \psi(t, y) \leq$ $\delta \lambda_{3} \phi(t, x), \forall t \in[0,1], x, y \in[0,+\infty) ;$

then we can get conclusions $(S 1)-(S 3)$ of Theorem 4.1 .

Proof The proof process is similar to that of Theorem 4.1.

Example 4.1 Consider the following problem:

$$
\left\{\begin{array}{l}
D_{0^{+}}^{\frac{13}{2}} x(t)+2(x+3)^{\frac{1}{4}}+3(x+2)^{\frac{1}{4}}+3(x+4)^{-\frac{1}{6}}+4(x+5)^{-\frac{1}{6}} \\
-\frac{22}{5} e^{-x}+4 e^{-y}+4 t+2 t^{2}+3 t^{3}+4 t^{4}+6=0, \quad 0<t<1, \\
x^{i}(0)=0, \quad 0 \leq i \leq 4, \quad D_{0^{+}}^{\frac{5}{3}} x(1)=\int_{0}^{\frac{3}{4}} x(s) \mathrm{d} s .
\end{array}\right.
$$

Here, $\alpha=\frac{13}{2} \in(5,6), \beta=\frac{5}{3} \in(1,2), \alpha-\beta-1=\frac{23}{6} \geq 0, \eta=\frac{3}{4}, \lambda_{1}=1, \lambda_{2}=2, \lambda_{3}=3, \lambda_{4}=4$, and

$$
\begin{aligned}
& f(t, x, y)=2(x+3)^{\frac{1}{4}}+3(y+4)^{-\frac{1}{6}}+6+4 t \\
& g(t, x, y)=-\frac{11}{5} e^{-x}+2 e^{-y}+3+t^{2} \\
& \phi(t, x)=(x+2)^{\frac{1}{4}}+t^{3} \\
& \psi(t, y)=(y+5)^{-\frac{1}{6}}+t^{4} .
\end{aligned}
$$

Thus, $\Gamma(\alpha+1)-\Gamma(\alpha-\beta) \eta^{\alpha}=\Gamma\left(\frac{15}{2}\right)-\Gamma\left(\frac{29}{6}\right) \frac{3}{4}^{\frac{13}{2}}=1.868 \times 10^{-3}, f(t, 0,1)=2 \cdot 3^{\frac{1}{4}}+3 \cdot 5^{-\frac{1}{5}}+$ $6+4 t \not \equiv 0, g(t, 0,1)=-\frac{11}{5}+2 e^{-1}+t^{2} \not \equiv 0, \phi(t, 0)=2^{\frac{1}{4}}+t^{3} \not \equiv 0, \psi(t, 1)=3 \times 6^{-\frac{1}{6}}+t^{4} \not \equiv 0$.

For $\forall \lambda \in(0,1), t \in[0,1], x, y \in[0,+\infty)$, there exists $\gamma=\frac{1}{4} \in(0,1)$, we can get that

$$
\begin{aligned}
f\left(t, \lambda x, \lambda^{-1} y\right) & =2(\lambda x+3)^{\frac{1}{4}}+3\left(\lambda^{-1} y+4\right)^{-\frac{1}{6}}+6+4 t \\
& \geq \lambda^{\frac{1}{4}} 2(x+3)^{\frac{1}{4}}+\lambda^{\frac{1}{6}} 3(y+4)^{-\frac{1}{5}}+6+4 t \\
& \geq \lambda^{\frac{1}{4}}\left(2(x+3)^{\frac{1}{4}}+3(y+4)^{-\frac{1}{6}}+6+4 t\right) \\
& =\lambda^{\frac{1}{4}} f(t, x, y) .
\end{aligned}
$$

For $\forall t \in[0,1], x, y \in[0,+\infty)$, there is

$$
g_{x x}^{\prime \prime}(t, x, y)=-\frac{11}{5} e^{-x} \leq 0, \quad g_{y y}^{\prime \prime}(t, x, y)=2 e^{-y} \geq 0,
$$


we obtain that, for fixed $t \in[0,1], y \in[0,+\infty), g(t, \cdot y)$ is concave; for fixed $t \in[0,1], x \in$ $[0,+\infty), g(t, x, \cdot)$ is convex. For $\forall \lambda, t \in[0,1], x, y \in[0,+\infty)$, we have

$$
\begin{aligned}
\phi(t, \lambda x) & =(\lambda x+2)^{\frac{1}{4}}+t^{3} \geq \lambda^{\frac{1}{4}}(x+2)^{\frac{1}{4}}+t^{3} \\
& \geq \lambda\left((\lambda x+2)^{\frac{1}{4}}+t^{3}\right)=\lambda \phi(t, x)
\end{aligned}
$$

and

$$
\psi\left(t, \lambda^{-1} y\right)=\left(\lambda^{-1} y+5\right)^{-\frac{1}{6}}+t^{4} \geq \lambda\left((y+5)^{-\frac{1}{6}}+t^{4}\right)=\lambda \psi(t, y)
$$

Let $l^{\prime}$ be a sufficiently large constant and $x, y \leq l^{\prime}, \widetilde{c}=\frac{1}{25}$, there is

$$
g\left(t, \theta, l^{\prime}\right)=-\frac{11}{5}+2 e^{-l^{\prime}}+3+t^{2} \geq \frac{1}{25}\left(-\frac{11}{5} e^{-l^{\prime}}+2+3+t^{2}\right)=\tilde{c} g\left(t, l^{\prime}, \theta\right) .
$$

Let $\delta=2$, by calculation, we have

$$
\begin{aligned}
& \lambda_{2} g(t, x, y)+\lambda_{3} \phi(t, x)+\lambda_{4} \psi(t, y) \\
& \quad=2\left[-\frac{11}{5} e^{-x}+2 e^{-y}+3+t^{2}\right]+3\left[(x+2)^{\frac{1}{4}}+t^{3}\right]+4\left[(y+5)^{-\frac{1}{6}}+t^{4}\right] \\
& \quad \leq 3(x+2)^{\frac{1}{4}}+4(y+5)^{-\frac{1}{6}}+6-\frac{22}{5} e^{-x}+4 e^{-y}+2 t^{2}+3 t^{3}+4 t^{4} \\
& \quad \leq 4(x+3)^{\frac{1}{4}}+6(y+4)^{-\frac{1}{6}}+12+8 t \\
& \quad \leq 2\left[2(x+2)^{\frac{1}{4}}+3(y+4)^{-\frac{1}{6}}+6+4 t\right] \\
& \quad=\delta \lambda_{1} f(t, x, y) .
\end{aligned}
$$

Therefore, all the assumptions of Theorem 4.1 are satisfied, then Example 4.1 has a unique positive solution $x^{*} \in P_{h}$, where $h(t)=t^{\frac{11}{2}}, t \in[0,1]$. Besides, the other condition of Theorem 4.1 holds.

Example 4.2 Consider the following problem:

$$
\left\{\begin{array}{c}
D_{0^{+}}^{\frac{7}{3}} x(t)+(x+3)^{\frac{1}{4}}+2(x+2)^{\frac{1}{4}}+(y+4)^{-\frac{1}{5}}+3(y+5)^{-\frac{1}{6}} \\
-\frac{11}{5} e^{-x}+2 e^{-y}+4 t+t^{2}+t^{3}+t^{4}+6=0, \quad 0<t<1, \\
x^{i}(0)=0, \quad 0 \leq i \leq 4, \quad D_{0^{+}}^{\frac{1}{4}} x(1)=\int_{0}^{\frac{3}{4}} x(s) \mathrm{d} s .
\end{array}\right.
$$

Here, $\alpha=\frac{7}{3} \in(2,3), \beta=\frac{1}{4} \in(0,1), \alpha-\beta-1=\frac{13}{12} \geq 0, \eta=\frac{1}{2}, \lambda_{1}=2, \lambda_{2}=1, \lambda_{3}=4, \lambda_{4}=3$, and

$$
\begin{aligned}
& f(t, x, y)=2(x+1)^{\frac{1}{2}}+\frac{1}{1+y}+\sin t, \\
& g(t, x, y)=-\frac{3}{4} e^{-x}+\frac{12}{5} e^{-y}+3+\cos t, \\
& \phi(t, x)=(x+4)^{\frac{1}{2}}+2,
\end{aligned}
$$




$$
\psi(t, y)=\frac{1}{1+y}+t^{2}
$$

Therefore, $\Gamma(\alpha+1)-\Gamma(\alpha-\beta) \eta^{\alpha}=\Gamma\left(\frac{10}{3}\right)-\Gamma\left(\frac{25}{12}\right) \frac{1}{2}^{\frac{7}{3}}=2.5722, f(t, 0,1)=2^{\frac{1}{2}}+\frac{1}{2}+\sin t$, $g(t, 0,1)=-\frac{3}{4}+\frac{12}{5} e^{-1}+\cos t, \phi(t, 0)=4^{\frac{1}{2}}+2, \psi(t, 1)=\frac{1}{2}+t^{2} \not \equiv 0$.

For $\forall \lambda \in(0,1), t \in[0,1], x, y \in[0,+\infty)$, we can derive that

$$
\begin{aligned}
f\left(t, \lambda x, \lambda^{-1} y\right) & =2(\lambda x+1)^{\frac{1}{2}}+\frac{1}{1+y}+\sin t \\
& \geq \lambda^{\frac{1}{2}} 2(x+1)^{\frac{1}{2}}+\lambda \frac{1}{1+y}+\sin t \\
& \geq \lambda\left(2(x+1)^{\frac{1}{2}}+\frac{1}{1+y}+\sin t\right) \\
& =\lambda f(t, x, y) .
\end{aligned}
$$

For expression of $g$, there is

$$
g_{x x}^{\prime \prime}(t, x, y)=-\frac{3}{4} e^{-x} \leq 0, \quad g_{y y}^{\prime \prime}(t, x, y)=\frac{12}{5} e^{-y} \geq 0,
$$

i.e., $g(t, \cdot, y)$ is concave, $g(t, x, \cdot)$ is convex. Further, there exist $\gamma=\frac{1}{2} \in(0,1)$,

$$
\phi(t, \lambda x)=(\lambda x+4)^{\frac{1}{2}}+2 \geq \lambda^{\frac{1}{2}}\left((x+2)^{\frac{1}{4}}+2\right)=\lambda^{\frac{1}{2}} \phi(t, x),
$$

and

$$
\psi\left(t, \lambda^{-1} y\right)=\frac{1}{1+\lambda^{-1} y}+t^{2} \geq \lambda\left(\frac{1}{1+y}+t^{2}\right)=\lambda \psi(t, y)
$$

Let $l^{\prime}$ be a sufficiently large constant with $x, y \leq l^{\prime} . c=\frac{1}{9}$, we can obtain

$$
g\left(t, \theta, l^{\prime}\right)=-\frac{3}{4}+\frac{12}{5} e^{-l^{\prime}}+3+\cos t \geq \frac{1}{9}\left(-\frac{3}{4} e^{-l^{\prime}}+\frac{12}{5}+3+\cos t\right)=c g\left(t, l^{\prime}, \theta\right) .
$$

Let $\delta=2.5$, we get

$$
\begin{array}{rl}
\lambda_{1} & f(t, x, y)+\lambda_{2} g(t, x, y)+\lambda_{4} \psi(t, y) \\
& =2\left[2(x+1)^{\frac{1}{2}}+\frac{1}{1+y}+\sin t\right]+\left[-\frac{3}{4} e^{-x}+\frac{12}{5} e^{-y}+3+\cos t\right]+3\left[\frac{1}{1+y}+t^{2}\right] \\
& =4(x+1)^{\frac{1}{2}}+\frac{5}{1+y}+2 \sin t-\frac{3}{4} e^{-x}+\frac{12}{5} e^{-y}+3+\cos t+3 t^{2} \\
& \leq 4(x+4)^{\frac{1}{2}}+17 \\
& \leq 2.5 \times 4\left[(x+4)^{\frac{1}{2}}+2\right]=\delta \lambda_{3} \phi(t, x) .
\end{array}
$$

Then Example 4.2 has a unique positive solution $x^{*} \in P_{h}$, where $h(t)=t^{\frac{4}{3}}, t \in[0,1]$. 


\section{Acknowledgements}

First and foremost, we would like to express our deepest gratitude to our supervisor, Dr. Zhang Lingling, who has provided us with valuable help in the writing of this paper. Then we shall extend many thanks to Mr. Zhou Bibo, Miss Tian Huimin for all their kindness and help. Last but not least, we would like to thank the opening project. This paper is supported by the opening project of State Key Laboratory of Explosion Science and Technology. This paper is also supported by Key Research and Development program of Shanxi Province.

\section{Funding}

The project is the opening project of State Key Laboratory of Explosion Science and Technology (Beijing Institute of Technology). The opening project number is KFJJ19-06M. Another project is Key Research and Development program of Shanxi Province (International Cooperation). The project number is 201903D421042.

\section{Availability of data and materials}

Not applicable.

\section{Competing interests}

The authors declare that they have no competing interests.

\section{Authors' contributions}

NZ and LZ carried out the concepts, design, definition of content, literature search, and manuscript preparation. NZ drafted the manuscript. BZ and HT performed manuscript review. All authors have read and approved the content of the manuscript.

\section{Publisher's Note}

Springer Nature remains neutral with regard to jurisdictional claims in published maps and institutional affiliations.

Received: 11 November 2019 Accepted: 26 February 2020 Published online: 11 March 2020

\section{References}

1. Zhai, C., Wang, W., Zhang, L.: Generalization for a class of concave and convex operators. Acta Math. Sin. 51, 529-540 (2008) (in Chinese)

2. Zhai, C., Zhang, L.: New fixed point theorems for mixed monotone operators and local existence-uniqueness of positive solutions for nonlinear boundary value problems. J. Math. Anal. Appl. 382, 594-614 (2011)

3. Zhai, C., Hao, M.: Fixed point theorems for mixed monotone operators with perturbation and applications to fractional differential equation boundary value problems. Nonlinear Anal. 75, 2542-2551 (2012)

4. Wang, H., Zhang, L.: The solution for a class of sum operator equation and its application to fractional differential equation boundary value problems. Bound. Value Probl. 2015, 1 (2015)

5. Zhang, X., Liu, L., Wu, Y.: Fixed point theorems for the sum of three classes of mixed monotone operators and applications. Fixed Point Theory Appl. 2016, 49 (2016)

6. Zhai, C., Wang, L.: $\varphi-(h, e)$-concave operators and applications. J. Math. Anal. Appl. 454, 571-584 (2017)

7. Zhang, L., Wang, H., Wang, X.: Fixed point results in set $P_{(\text {he })}$ with applications to fractional differential equations. Topol. Methods Nonlinear Anal. 54(2), 537-566 (2019)

8. Guo, D., Lakshmikantham, V.: Coupled fixed points of nonlinear operators with applications. Nonlinear Anal. 11 623-632 (1987)

9. Guo, D., Lakshmikantham, V.: Nonlinear Problems in Abstract Cones. Academic Press, San Diego (1988)

10. Podlubny, I.: Fractional Differential Equations. Mathematics in Science and Engineering. Academic Press, New York (1999)

11. Amann, $H_{\text {.: }}$ On the number of solutions of nonlinear equations in ordered Banach spaces. J. Funct. Anal. 11, 346-384 (1972)

12. Kilbas, A.A., Srivastava, H.M., Trujillo, J.J.: Theory and Applications of Fractional Differential Equations. Elsevier, Amsterdam (2006)

13. Li, S., Liu, Z.: Perturbations from symmetric elliptic boundary value problems. J. Differ. Equ. 185, 271-280 (2002)

14. Zhang, X.: Existence and iteration of monotone positive solutions for an elastic beam with a corner. Nonlinear Anal. 10, 2097-2103 (2009)

15. Zhai, C., Yang, C., Guo, C.: Positive solutions of operator equation on ordered Banach spaces and applications. Comput. Math. Appl. 56, 3150-3156 (2008)

16. Zhang, C., Sun, S.: Sturm-Picone comparison theorem of a kind of conformable fractional differential equations on time scales. J. Appl. Math. Comput. 55, 191-203 (2017)

17. Yang, W.: Monotone iterative technique for a coupled system of nonlinear Hadamard fractional differential equations. J. Appl. Math. Comput. 59, 585-596 (2019)

18. Liu, X., Jia, M.: The method of lower and upper solutions for the general boundary value problems of fractional differential equations with p-Laplacian. Adv. Differ. Equ. 2018, 28 (2018)

19. Rao, S.N., Alesemi, M.: On a coupled system of fractional differential equations with nonlocal non-separated boundary conditions. Adv. Differ. Equ. 2019, 97 (2019)

20. Ge, F., Kou, C.: Stability analysis by Krasnoselskii's fixed point theorem for nonlinear fractional differential equations. Appl. Math. Comput. 257, 308-316 (2015)

21. Zhang, L., Tian, H.: Existence and uniqueness of positive solutions for a class of nonlinear fractional differential equations. Adv. Differ. Equ. 2017, 114 (2017)

22. Cabada, A., Hamdi, Z.: Nonlinear fractional differential equations with integral boundary value conditions. Appl. Math. Comput. 228, 251-257 (2014) 
23. Cabada, A., Dimitrijevic, S., Tomovic, T., Aleksic, S.: The existence of a positive solution for nonlinear fractional differential equations with integral boundary value conditions. Math. Methods Appl. Sci. 40(6), 1880-1891 (2017)

24. Xu, X.J., Fei, X.L.: The positive properties of Green's function for three point boundary value problems of nonlinear fractional differential equations and its applications. Commun. Nonlinear Sci. Numer. Simul. 17, 1555-1565 (2012)

25. Wang, Y.P., Sun, S.R.: Solvability to infinite-point boundary value problems for singular fractional differential equations on the half-line. J. Appl. Math. Comput. 57, 359-373 (2018)

Submit your manuscript to a SpringerOpen ${ }^{\circ}$ journal and benefit from:

- Convenient online submission

- Rigorous peer review

- Open access: articles freely available online

- High visibility within the field

- Retaining the copyright to your article

Submit your next manuscript at $\boldsymbol{\triangleright}$ springeropen.com 